\title{
Multilevel Picard iterations for solving smooth semilinear parabolic heat equations
}

\author{
Weinan $E^{1}(D) \cdot$ Martin Hutzenthaler ${ }^{2}$. Arnulf Jentzen ${ }^{3,4,5}$ (D) Thomas Kruse $^{6}$
}

Received: 11 December 2019 / Accepted: 18 March 2021 / Published online: 4 November 2021

(c) The Author(s) 2021

\begin{abstract}
We introduce a new family of numerical algorithms for approximating solutions of general high-dimensional semilinear parabolic partial differential equations at single space-time points. The algorithm is obtained through a delicate combination of the Feynman-Kac and the Bismut-Elworthy-Li formulas, and an approximate decomposition of the Picard fixed-point iteration with multilevel accuracy. The algorithm has been tested on a variety of semilinear partial differential equations that arise in physics and finance, with satisfactory results. Analytical tools needed for the analysis of such algorithms, including a semilinear Feynman-Kac formula, a new class of seminorms and their recursive inequalities, are also introduced. They allow us to prove for semilinear heat equations with gradient-independent nonlinearities that the computational complexity of the proposed algorithm is bounded by $O\left(d \varepsilon^{-(4+\delta)}\right)$ for any $\delta \in(0, \infty)$ under suitable assumptions, where $d \in \mathbb{N}$ is the dimensionality of the problem and $\varepsilon \in(0, \infty)$ is the prescribed accuracy. Moreover, the introduced class of numerical algorithms is also powerful for proving high-dimensional approximation capacities for deep neural networks.
\end{abstract}

Keywords Curse of dimensionality $\cdot$ High-dimensional PDEs $\cdot$ High-dimensional semilinear BSDEs · Multilevel Picard iteration · Multilevel Monte Carlo method

\section{Mathematics Subject Classification 65M75}

\section{Introduction and main results}

High-dimensional partial differential equations (PDEs) arise naturally in many important areas including quantum mechanics, statistical physics, financial engineering, economics, etc. Yet developing efficient and practical algorithms for these high-dimensional PDEs has been a long-standing problem and indeed one of the most challenging tasks in mathematics.

This article is part of the topical collection "Deep learning and PDEs" edited by Arnulf Jentzen, Lin Lin, Siddhartha Mishra, and Lexing Ying.

Arnulf Jentzen

ajentzen@uni-muenster.de; ajentzen@cuhk.edu.cn

Extended author information available on the last page of the article 
The difficulty lies in the "curse of dimensionality" [5], i.e., the complexity of the problem goes up exponentially as a function of dimension, which is a well-known obstacle that is also at the heart of many other important subjects such as high-dimensional statistics and the modeling of many-body systems.

For linear parabolic PDEs, the Feynman-Kac formula establishes an explicit representation of the solution of the PDE as the expectation of the solution of an appropriate stochastic differential equation (SDE). Monte Carlo methods together with suitable discretizations of the SDE (see, e.g., $[30,31,38,39]$ ) then allow to approximate the solution at any single point in space-time with a computational complexity that grows as $O\left(d \varepsilon^{-(2+\delta)}\right)$ for any $\delta \in(0, \infty)$ where $d$ is the dimensionality of the problem and $\varepsilon$ is the accuracy required (cf., e.g., $[20,23,25,26])$.

In the seminal papers [40-42], Pardoux \& Peng established a generalized nonlinear Feynman-Kac formula that gives an explicit representation of the solution of a semilinear parabolic PDE through the solution of an appropriate backward stochastic differential equation (BSDE). Solving BSDEs numerically, however, requires in general suitable discretizations of nested conditional expectations (see, e.g., $[8,47]$ ) and the straightforward Monte Carlo method applied to these nested conditional expectations results in an algorithm with a computational complexity that grows polynomially in $d$ but at least exponentially in $\varepsilon^{-1}$. Other discretization methods for the nested conditional expectations proposed in the literature include the quantization tree method (see [3]), the regression method based on Malliavin calculus or based on kernel estimation (see [8]), the projection on function spaces method (see [22]), the cubature on Wiener space method (see [13]), and the Wiener chaos decomposition method (see [9]). None of these algorithms meets the requirement that the computational complexity has been proven to grow at most polynomially both in $d$ and $\varepsilon^{-1}$ (see [17, Sects. 6.1-6.6] for a detailed discussion of these approximation methods).

Another probabilistic representation for the solutions of some semilinear parabolic PDEs with polynomial nonlinearity has been established in Skorohod [45] by means of branching diffusion processes. Recently this classical representation has been extended to more general analytic nonlinearities [27-29]. This probabilistic representation has been successfully used to obtain a Monte Carlo approximation method for semilinear parabolic PDEs with a computational complexity that grows polynomially both in $d$ and $\varepsilon^{-1}$. However, this method is only applicable to PDEs with analytic nonlinearities and it requires the terminal/initial condition to be suitably small (see [17, Sect. 6.7] for a detailed discussion).

In this paper we propose a new family of numerical algorithms for approximating solutions of general high-dimensional semilinear parabolic PDEs (and BSDEs) at single space-time points; see(12) below for the definition of our approximations. For semilinear heat equations with gradient-independent nonlinearities we prove that the computational complexity (see Corollary 3.19 below for the precise meaning hereof) of our proposed algorithm is $O\left(d \varepsilon^{-(4+\delta)}\right)$ for any $\delta \in(0, \infty)$. For this we assume that the PDE solution $u:[0, T] \times \mathbb{R}^{d} \rightarrow \mathbb{R}$ is infinitely often differentiable and that the nonlinearity and the rescaled derivatives $(k !)^{-\frac{3}{4}}\left(\frac{\partial}{\partial t}+\frac{1}{2} \Delta_{x}\right)^{k} u$ are uniformly bounded in time, space, $k \in \mathbb{N}_{0}$, and in the dimension; see Corollary 3.19 below for details. In Sect. 3.8 we provide an example which satisfies these assumptions. These strong smoothness assumptions are used in the error analysis in this article.

After a first preprint of this work has appeared, a series of further research articles inspired by this work have appeared; see, e.g., [4,17,32-35]. In particular, the paper [35] analyzes the algorithm(12) in the case of gradient-dependent nonlinearities under stronger smoothness assumptions than those of Corollary 3.19. Moreover, the paper [33] relaxes 
the smoothness assumptions of Corollary 3.19 and proves for general Lipschitz continuous, gradient-independent nonlinearities that a variant of the algorithm(12) with quadrature rules being replaced by Monte Carlo averages is efficient in high dimensions. Furthermore, in [32] this variant of the algorithm (12) is used to prove that deep neural networks overcome the curse of dimensionality in the numerical approximation of solutions of semilinear heat equations with gradient-independent nonlinearities.

The algorithm (12), which we will call "multilevel Picard iteration", is a delicate combination of the Feynman-Kac and Bismut-Elworthy-Li formulas, and a decomposition of the Picard iteration with multilevels of accuracy. The efficiency and accuracy of the proposed algorithm has been tested on a variety of semilinear parabolic PDEs that arise in physics and finance. These details are presented in [17]. To get a feeling about the performance of the algorithm: To approximate $u(1,0)$ for the solution $u:[0,1] \times \mathbb{R}^{d} \rightarrow \mathbb{R}$ of

$$
\partial_{t} u=\frac{1}{2} \Delta u+u-u^{3}
$$

with $d=100, \varepsilon=0.01, u(0, x)=\left(1+\max \left\{\left|x_{1}\right|^{2}, \ldots,\left|x_{100}\right|^{2}\right\}\right)^{-1}$ requires $10 \mathrm{~s}$ of runtime on a $2.8 \mathrm{GHz}$ Intel i7 processor with $16 \mathrm{~GB}$ RAM.

We also introduce the tools needed to analyze these high-dimensional algorithms. Some of these tools are quite non-standard (e.g. the seminorms (19) and the recursive inequality (53) involving different seminorms). Using these tools, we are able to rigorously prove the bounds for the computational complexity mentioned above.

\subsection{Notation}

Since the proposed algorithm relies heavily on the Feynman-Kac formula, we will adopt the notations and conventions in stochastic analysis. In addition, we frequently use the following notation. We denote by $\|\cdot\|:\left(\cup_{n \in \mathbb{N}} \mathbb{R}^{n}\right) \rightarrow[0, \infty)$ and by $\langle\cdot, \cdot\rangle:\left(\cup_{n \in \mathbb{N}} \mathbb{R}^{n} \times \mathbb{R}^{n}\right) \rightarrow$ $[0, \infty)$ the functions which satisfy for all $n \in \mathbb{N}, v=\left(v_{1}, \ldots, v_{n}\right), w=\left(w_{1}, \ldots, w_{n}\right) \in \mathbb{R}^{n}$ that $\|v\|=\left[\sum_{i=1}^{n}\left|v_{i}\right|^{2}\right]^{1 / 2}$ and $\langle v, w\rangle=\sum_{i=1}^{n} v_{i} w_{i}$. For every topological space $(E, \mathcal{E})$ we denote by $\mathcal{B}(E)$ the Borel-sigma-algebra on $(E, \mathcal{E})$. For all measurable spaces $(A, \mathcal{A})$ and $(B, \mathcal{B})$ we denote by $\mathcal{M}(\mathcal{A}, \mathcal{B})$ the set of $\mathcal{A} / \mathcal{B}$-measurable functions from $A$ to $B$. For all metric spaces $\left(E, d_{E}\right)$ and $\left(F, d_{F}\right)$ we denote by $\operatorname{Lip}(E, F)$ the set of all globally Lipschitz continuous functions from $E$ to $F$. For every $d \in \mathbb{N}$ we denote by $\mathbb{R}_{\text {Inv }}^{d \times d}$ the set of invertible matrices in $\mathbb{R}^{d \times d}$. For every $d \in \mathbb{N}$ and every $A \in \mathbb{R}^{d \times d}$ we denote by $A^{*} \in \mathbb{R}^{d \times d}$ the transpose of $A$. For every $d \in \mathbb{N}$ and every $x=\left(x_{1}, \ldots, x_{d}\right) \in \mathbb{R}^{d}$ we denote by $\operatorname{diag}(x) \in \mathbb{R}^{d \times d}$ the diagonal matrix with diagonal entries $x_{1}, \ldots, x_{d}$. For every $T \in(0, \infty)$ we denote by $\mathcal{Q}_{T}$ the set given by $\mathcal{Q}_{T}=\left\{w:[0, T] \rightarrow \mathbb{R}: w^{-1}(\mathbb{R} \backslash\{0\})\right.$ is a finite set $\}$. We denote by $\lfloor\cdot]: \mathbb{R} \rightarrow \mathbb{Z}$ and $[\cdot]^{+}: \mathbb{R} \rightarrow[0, \infty)$ the functions that satisfy for all $x \in \mathbb{R}$ that $\lfloor x\rfloor=\max (\mathbb{Z} \cap(-\infty, x])$ and $[x]^{+}=\max \{x, 0\}$. We use the conventions $0 \cdot \infty=0$ and $0^{0}=1$.

\section{Multilevel Picard iteration for semilinear parabolic PDEs}

\subsection{A fixed-point equation for semilinear PDEs}

Let $T \in(0, \infty), d \in \mathbb{N}$, let $g: \mathbb{R}^{d} \rightarrow \mathbb{R}, f:[0, T] \times \mathbb{R}^{d} \times \mathbb{R} \times \mathbb{R}^{d} \rightarrow \mathbb{R}, u:[0, T] \times \mathbb{R}^{d} \rightarrow$ $\mathbb{R}, \mu:[0, T] \times \mathbb{R}^{d} \rightarrow \mathbb{R}^{d}$, and $\sigma=\left(\sigma_{1}, \ldots, \sigma_{d}\right):[0, T] \times \mathbb{R}^{d} \rightarrow \mathbb{R}_{\text {Inv }}^{d \times d}$ be sufficiently regular functions, assume for all $t \in[0, T), x \in \mathbb{R}^{d}$ that $u(T, x)=g(x)$ and 


$$
\begin{aligned}
& \partial_{t} u(t, x)+f\left(t, x, u(t, x),[\sigma(t, x)]^{*} \nabla_{x} u(t, x)\right)+\left\langle\mu(t, x), \nabla_{x} u(t, x)\right\rangle \\
& \quad+\frac{1}{2} \operatorname{Trace}\left(\sigma(t, x)[\sigma(t, x)]^{*} \operatorname{Hess}_{x} u(t, x)\right)=0,
\end{aligned}
$$

let $\left(\Omega, \mathcal{F}, \mathbb{P},\left(\mathbb{F}_{t}\right)_{t \in[0, T]}\right)$ be a stochastic basis (cf., e.g., [43, Appendix E]), let $W=$ $\left(W^{1}, \ldots, W^{d}\right):[0, T] \times \Omega \rightarrow \mathbb{R}^{d}$ be a standard $\left(\mathbb{F}_{t}\right)_{t \in[0, T]}$-Brownian motion, and for every $s \in[0, T], x \in \mathbb{R}^{d}$ let $X^{s, x}:[s, T] \times \Omega \rightarrow \mathbb{R}^{d}$ and $D^{s, x}:[s, T] \times \Omega \rightarrow \mathbb{R}^{d \times d}$ be

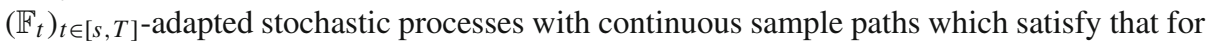
all $t \in[s, T]$ it holds $\mathbb{P}$-a.s. that

$$
\begin{aligned}
& X_{t}^{s, x}=x+\int_{s}^{t} \mu\left(r, X_{r}^{s, x}\right) d r+\sum_{j=1}^{d} \int_{s}^{t} \sigma_{j}\left(r, X_{r}^{s, x}\right) d W_{r}^{j}, \\
& D_{t}^{s, x}=\mathrm{I}_{\mathbb{R}^{d \times d}}+\int_{s}^{t}\left(\frac{\partial}{\partial x} \mu\right)\left(r, X_{r}^{s, x}\right) D_{r}^{s, x} d r+\sum_{j=1}^{d} \int_{s}^{t}\left(\frac{\partial}{\partial x} \sigma_{j}\right)\left(r, X_{r}^{s, x}\right) D_{r}^{s, x} d W_{r}^{j}
\end{aligned}
$$

(cf., e.g., [36, Chapter 5], [24], or [2] for existence and uniqueness results for stochastic differential equations of the form (3)). For every $s \in[0, T]$ the processes $D^{s, x}, x \in \mathbb{R}^{d}$, are in a suitable sense the derivative processes of $X^{s, x}, x \in \mathbb{R}^{d}$, with respect to $x \in \mathbb{R}^{d}$. Using the Feynman-Kac formula (cf., e.g., [36, Theorem 5.7.6]), we have from (2) for all $(s, x) \in[0, T] \times \mathbb{R}^{d}$ that

$$
u(s, x)=\mathbb{E}\left[g\left(X_{T}^{s, x}\right)\right]+\int_{s}^{T} \mathbb{E}\left[f\left(t, X_{t}^{s, x}, u\left(t, X_{t}^{s, x}\right),\left[\sigma\left(t, X_{t}^{s, x}\right)\right]^{*}\left(\nabla_{x} u\right)\left(t, X_{t}^{s, x}\right)\right)\right] d t
$$

In (4) the derivative of $u$ appears on the right-hand side and, therefore, (4) does not provide a closed fixed point equation. To obtain such a closed fixed point equation we now bring the Bismut-Elworthy-Li formula into play (see, e.g., Elworthy \& Li [18, Theorem 2.1] or Da Prato \& Zabczyk [14, Theorem 2.1]). This gives us for all $(s, x) \in[0, T) \times \mathbb{R}^{d}$

$$
\begin{gathered}
{[\sigma(s, x)]^{*}\left(\nabla_{x} u\right)(s, x)=\mathbb{E}\left[g\left(X_{T}^{s, x}\right) \frac{[\sigma(s, x)]^{*}}{T-s} \int_{s}^{T}\left[\sigma\left(r, X_{r}^{s, x}\right)^{-1} D_{r}^{s, x}\right]^{*} d W_{r}\right]} \\
+\int_{s}^{T} \mathbb{E}\left[f\left(t, X_{t}^{s, x}, u\left(t, X_{t}^{s, x}\right),\left[\sigma\left(t, X_{t}^{s, x}\right)\right]^{*}\left(\nabla_{x} u\right)\left(t, X_{t}^{s, x}\right)\right)\right. \\
\left.\cdot \frac{[\sigma(s, x)]^{*}}{t-s} \int_{s}^{t}\left[\sigma\left(r, X_{r}^{s, x}\right)^{-1} D_{r}^{s, x}\right]^{*} d W_{r}\right] d t .
\end{gathered}
$$

Now let $\mathbf{u}^{\infty} \in \operatorname{Lip}\left([0, T) \times \mathbb{R}^{d}, \mathbb{R}^{1+d}\right)$ be defined by $\mathbf{u}^{\infty}(s, x)=\left(u(s, x),[\sigma(s, x)]^{*}\right.$ $\left.\left(\nabla_{x} u\right)(s, x)\right)$ for all $(s, x) \in[0, T) \times \mathbb{R}^{d}$. Let $\boldsymbol{\Phi}: \operatorname{Lip}\left([0, T) \times \mathbb{R}^{d}, \mathbb{R}^{1+d}\right) \rightarrow \operatorname{Lip}([0, T) \times$ $\mathbb{R}^{d}, \mathbb{R}^{1+d}$ ) be defined by

$$
\begin{aligned}
(\boldsymbol{\Phi}(\mathbf{v}))(s, x)= & \mathbb{E}\left[g\left(X_{T}^{s, x}\right)\left(1, \frac{[\sigma(s, x)]^{*}}{T-s} \int_{s}^{T}\left[\sigma\left(r, X_{r}^{s, x}\right)^{-1} D_{r}^{s, x}\right]^{*} d W_{r}\right)\right] \\
& +\int_{s}^{T} \mathbb{E}\left[f\left(t, X_{t}^{s, x}, \mathbf{v}\left(t, X_{t}^{s, x}\right)\right)\left(1, \frac{[\sigma(s, x)]^{*}}{t-s} \int_{s}^{t}\left[\sigma\left(r, X_{r}^{s, x}\right)^{-1} D_{r}^{s, x}\right]^{*} d W_{r}\right)\right] d t
\end{aligned}
$$

for all $\mathbf{v} \in \operatorname{Lip}\left([0, T) \times \mathbb{R}^{d}, \mathbb{R}^{1+d}\right),(s, x) \in[0, T) \times \mathbb{R}^{d}$. Combining (6) with (4) and (5) gives

$$
\mathbf{u}^{\infty}=\boldsymbol{\Phi}\left(\mathbf{u}^{\infty}\right) .
$$


Next we define a sequence of Picard iterations $\left(\mathbf{u}_{k}\right)_{k \in \mathbb{N}_{0}} \subseteq \operatorname{Lip}\left([0, T) \times \mathbb{R}^{d}, \mathbb{R}^{1+d}\right)$ associated to (6),

$$
\mathbf{u}_{k}(s, x)=\left(\boldsymbol{\Phi}\left(\mathbf{u}_{k-1}\right)\right)(s, x)
$$

for all $k \in \mathbb{N}, s \in[0, T), x \in \mathbb{R}^{d}$. This sequence of Picard iterations has already been studied in the literature; see, e.g., [46, Thereom 7.3.4] or [6]. Under suitable assumptions, e.g., [46, Thereom 7.3.4] ensures that for all $s \in[0, T), x \in \mathbb{R}^{d}$ it holds that $\lim _{k \rightarrow \infty} \mathbf{u}_{k}(s, x)=$ $\mathbf{u}^{\infty}(s, x)$. Observe that for all $k \in \mathbb{N}, s \in[0, T), x \in \mathbb{R}^{d}$ it holds that

$$
\begin{aligned}
\mathbf{u}_{k}(s, x)= & \mathbf{u}_{1}(s, x)+\sum_{l=1}^{k-1}\left[\mathbf{u}_{l+1}(s, x)-\mathbf{u}_{l}(s, x)\right] \\
= & \left(\boldsymbol{\Phi}\left(\mathbf{u}_{0}\right)\right)(s, x)+\sum_{l=1}^{k-1}\left[\left(\boldsymbol{\Phi}\left(\mathbf{u}_{l}\right)\right)(s, x)-\left(\boldsymbol{\Phi}\left(\mathbf{u}_{l-1}\right)\right)(s, x)\right] \\
= & \mathbb{E}\left[g\left(X_{T}^{s, x}\right)\left(1, \frac{[\sigma(s, x)]^{*}}{T-s} \int_{s}^{T}\left[\sigma\left(r, X_{r}^{s, x}\right)^{-1} D_{r}^{s, x}\right]^{*} d W_{r}\right)\right] \\
& +\sum_{l=0}^{k-1} \int_{s}^{T} \mathbb{E}\left[\left(f\left(t, X_{t}^{s, x}, \mathbf{u}_{l}\left(t, X_{t}^{s, x}\right)\right)-\mathbb{1}_{\mathbb{N}}(l) f\left(t, X_{t}^{s, x}, \mathbf{u}_{l-1}\left(t, X_{t}^{s, x}\right)\right)\right)\right. \\
& \left.\cdot\left(1, \frac{[\sigma(s, x)]^{*}}{t-s} \int_{s}^{t}\left[\sigma\left(r, X_{r}^{s, x}\right)^{-1} D_{r}^{s, x}\right]^{*} d W_{r}\right)\right] d t .
\end{aligned}
$$

Next we incorporate a zero expectation term to slightly reduce the variance when approximating the expectation involving $g$ by Monte Carlo approximations. More precisely, for all $k \in \mathbb{N}, s \in[0, T), x \in \mathbb{R}^{d}$ it holds that

$$
\begin{gathered}
\mathbf{u}_{k}(s, x)=(g(x), 0)+\mathbb{E}\left[\left(g\left(X_{T}^{s, x}\right)-g(x)\right)\left(1, \frac{[\sigma(s, x)]^{*}}{T-s} \int_{s}^{T}\left[\sigma\left(r, X_{r}^{s, x}\right)^{-1} D_{r}^{s, x}\right]^{*} d W_{r}\right)\right] \\
+\sum_{l=0}^{k-1} \int_{s}^{T} \mathbb{E}\left[\left(f\left(t, X_{t}^{s, x}, \mathbf{u}_{l}\left(t, X_{t}^{s, x}\right)\right)-\mathbb{1}_{\mathbb{N}}(l) f\left(t, X_{t}^{s, x}, \mathbf{u}_{l-1}\left(t, X_{t}^{s, x}\right)\right)\right)\right. \\
\left.\cdot\left(1, \frac{[\sigma(s, x)]^{*}}{t-s} \int_{s}^{t}\left[\sigma\left(r, X_{r}^{s, x}\right)^{-1} D_{r}^{s, x}\right]^{*} d W_{r}\right)\right] d t .
\end{gathered}
$$

In this telescope expansion, we will apply a fundamental idea of Heinrich [25,26] and Giles [19] (control variates were also used, e.g., in [21,37]) and approximate the continuous quantities (expectation and time integral) by discrete ones (Monte Carlo averages and quadrature formulas respectively) with different degrees of accuracy at different levels of the Picard iteration. Since for large $l \in \mathbb{N}$ the difference between $\mathbf{u}_{l}$ and $\mathbf{u}_{l-1}$ is small, say $\rho^{-l}$ for some $\rho \in \mathbb{N}$, it suffices to approximate the expectation and the time integral with lower accuracy, say $\rho^{-(k-l)}$, at level $l \in\{0, \ldots, k-1\}$ for the $k$-th approximation to achieve a total error roughly of order $\rho^{-k}$. More precisely, we denote by $\left(q_{s}^{k, \rho}\right)_{k \in \mathbb{N}_{0}, \rho \in \mathbb{N}, s \in[0, T)} \subseteq \mathcal{Q}_{T}$ a family of quadrature formulas on $C([0, T], \mathbb{R})$ that we employ to approximate the time integrals $\int_{s}^{T} \ldots d t, s \in[0, T]$, appearing on the right-hand side of (10). We denote by $\Theta=\cup_{n \in \mathbb{N}} \mathbb{R}^{n}$ a set that allows to index families of independent random variables which we need for the Monte Carlo approximations. We denote by $\left(\mathfrak{m}_{k, \rho}\right)_{k \in \mathbb{N}_{0}, \rho \in \mathbb{N}}$ and $\left(m_{k, \rho}\right)_{k \in \mathbb{N}_{0}, \rho \in \mathbb{N}} \subseteq \mathbb{N}$ families of natural numbers that specify the number of Monte Carlo samples for approximating the expectations involving $g$ and $f$ on the right-hand side of (10). In Sect.3.1 we will take $\mathfrak{m}_{k, \rho}=m_{k, \rho}=\rho^{k}$ for every $k \in \mathbb{N}_{0}, \rho \in \mathbb{N}$ and we take $q^{k, \rho}$ as the Gauß-Legendre quadrature rule with $\rho$ nodes. Furthermore, for every $k \in \mathbb{N}_{0}, \rho \in \mathbb{N}$, 
$\theta \in \Theta,(s, x) \in[0, T) \times \mathbb{R}^{d}$ we denote by $\left(\mathcal{X}_{k, \rho}^{\theta}(s, x, t)\right)_{t \in[s, T]}$ and $\left(\mathcal{I}_{k, \rho}^{\theta}(s, x, t)\right)_{t \in(s, T]}$ the stochastic processes that we employ to approximate the processes $\left(X_{t}^{s, x}\right)_{t \in[s, T]}$ and $\left(1, \frac{[\sigma(s, x)]^{*}}{t-s} \int_{s}^{t}\left[\sigma\left(r, X_{r}^{s, x}\right)^{-1} D_{r}^{s, x}\right]^{*} d W_{r}\right)_{t \in(s, T]}$. More specifically, we choose for every $k \in \mathbb{N}_{0}, \rho \in \mathbb{N}, \theta \in \Theta,(s, x) \in[0, T) \times \mathbb{R}^{d}$ the processes $\left(\mathcal{X}_{k, \rho}^{\theta}(s, x, t)\right)_{t \in[s, T]}$ and $\left(\mathcal{I}_{k, \rho}^{\theta}(s, x, t)\right)_{t \in(s, T]}$ such that for all $t \in(s, T]$ we have that

$$
\begin{aligned}
\mathcal{X}_{k, \rho}^{\theta}(s, x, t) & \approx X_{t}^{s, x}, \\
\mathcal{I}_{k, \rho}^{\theta}(s, x, t) & \approx\left(1, \frac{[\sigma(s, x)]^{*}}{t-s} \int_{s}^{t}\left[\sigma\left(r, X_{r}^{s, x}\right)^{-1} D_{r}^{s, x}\right]^{*} d W_{r}\right) .
\end{aligned}
$$

For all these approximations the parameters $k \in \mathbb{N}_{0}, \rho \in \mathbb{N}$ specify the degree of discretization in the sense that the approximation errors get smaller in a suitable sense as $k \in \mathbb{N}_{0}$, $\rho \in \mathbb{N}$ get larger. We note that if we can sample from the distributions of the random variables on the right-hand sides of (11) as, e.g., in the case of Brownian motion, then we do not need to approximate them (see Example 2.1 below).

\subsection{The approximation scheme}

Let $T \in(0, \infty), d \in \mathbb{N}, \Theta=\cup_{n \in \mathbb{N}} \mathbb{R}^{n}$, let $g: \mathbb{R}^{d} \rightarrow \mathbb{R}, f:[0, T] \times \mathbb{R}^{d} \times \mathbb{R} \times \mathbb{R}^{d}$ $\rightarrow \mathbb{R}, \mu:[0, T] \times \mathbb{R}^{d} \rightarrow \mathbb{R}^{d}, \sigma:[0, T] \times \mathbb{R}^{d} \rightarrow \mathbb{R}_{\text {Inv }}^{d \times d}$ be measurable functions, let $\left(q_{s}^{k, \rho}\right)_{k \in \mathbb{N}_{0}, \rho \in \mathbb{N}, s \in[0, T)} \subseteq \mathcal{Q}_{T},\left(\mathfrak{m}_{k, \rho}\right)_{k \in \mathbb{N}_{0}, \rho \in \mathbb{N}},\left(m_{k, \rho}\right)_{k \in \mathbb{N}_{0}, \rho \in \mathbb{N}} \subseteq \mathbb{N}$, let $\left(\Omega, \mathcal{F}, \mathbb{P},\left(\mathbb{F}_{t}\right)_{t \in[0, T]}\right)$ be a stochastic basis, for every $l, \rho \in \mathbb{N}, \theta \in \Theta, x \in \mathbb{R}^{d}, s \in[0, T)$, $t \in[s, T]$ let $\mathcal{X}_{l, \rho}^{\theta}(s, x, t): \Omega \rightarrow \mathbb{R}^{d}$ and $\mathcal{I}_{l, \rho}^{\theta}(s, x, t): \Omega \rightarrow \mathbb{R}^{1+d}$ be functions, and for every $\theta \in \Theta, \rho \in \mathbb{N}$ let $\mathbf{U}_{k, \rho}^{\theta}:[0, T] \times \mathbb{R}^{d} \times \Omega \rightarrow \mathbb{R}^{d+1}, k \in \mathbb{N}_{0}$, be functions that satisfy for all $k \in \mathbb{N},(s, x) \in[0, T) \times \mathbb{R}^{d}$ that

$$
\begin{aligned}
& \mathbf{U}_{k, \rho}^{\theta}(s, x)=(g(x), 0)+\sum_{i=1}^{\mathfrak{m}_{k, \rho}} \frac{1}{\mathfrak{m}_{k, \rho}}\left[g\left(\mathcal{X}_{k, \rho}^{(\theta, 0,-i)}(s, x, T)\right)-g(x)\right] \mathcal{I}_{k, \rho}^{(\theta, 0,-i)}(s, x, T) \\
& \quad+\sum_{l=0}^{k-1} \sum_{i=1}^{m_{k-l, \rho}} \sum_{t \in[s, T]} \frac{q_{s}^{k-l, \rho}(t)}{m_{k-l, \rho}}\left[f\left(t, \mathcal{X}_{k-l, \rho}^{(\theta, l, i)}(s, x, t), \mathbf{U}_{l, \rho}^{(\theta, l, i, t)}\left(t, \mathcal{X}_{k-l, \rho}^{(\theta, l, i)}(s, x, t)\right)\right)\right. \\
& \left.-\mathbb{1}_{\mathbb{N}}(l) f\left(t, \mathcal{X}_{k-l, \rho}^{(\theta, l, i)}(s, x, t), \mathbf{U}_{[l-1]^{+}, \rho}^{(\theta,-l, i, t)}\left(t, \mathcal{X}_{k-l, \rho}^{(\theta, l, i)}(s, x, t)\right)\right)\right] \mathcal{I}_{k-l, \rho}^{(\theta, l, i)}(s, x, t) .
\end{aligned}
$$

Observe that the approximation scheme (12) employs Picard fixed-point iteration (cf., e.g., [6]), multilevel/multigrid techniques (see, e.g., [12,20,25,26]), discretizations of the SDE system (3), as well as quadrature approximations for the time integrals. The numerical approximations (12) are full history recursive in the sense that for every $(k, \rho) \in \mathbb{N} \times \mathbb{N}$ the full history $\mathbf{U}_{0, \rho}^{(\cdot)}, \mathbf{U}_{1, \rho}^{(\cdot)}, \ldots, \mathbf{U}_{k-1, \rho}^{(\cdot)}$ needs to be computed recursively in order to compute $\mathbf{U}_{k, \rho}^{(\cdot)}$. In this sense the numerical approximations (12) are full history recursive multilevel Picard approximations.

\subsection{Special case: semilinear heat equations}

In this subsection we specialize the numerical scheme(12) to the case of semilinear heat equations. 
Example 2.1 Assume the setting in Sect.2.2, let $W^{\theta}:[0, T] \times \Omega \rightarrow \mathbb{R}^{d}, \theta \in \Theta$, be independent standard $\left(\mathcal{F}_{t}\right)_{t \in[0, T]}$-Brownian motions, and assume for all $k \in \mathbb{N}_{0}, \rho \in \mathbb{N}$, $\theta \in \Theta, x \in \mathbb{R}^{d}, s \in[0, T), t \in[s, T], u \in(s, T]$ that $\mathcal{X}_{k, \rho}^{\theta}(s, x, t)=x+W_{t}^{\theta}-W_{s}^{\theta}$, $\mathcal{I}_{k, \rho}^{\theta}(s, x, s)=0, \mathcal{I}_{k, \rho}^{\theta}(s, x, u)=\left(1, \frac{W_{u}^{\theta}-W_{s}^{\theta}}{u-s}\right)$. Then it holds for all $\theta \in \Theta, k \in \mathbb{N}, \rho \in \mathbb{N}$, $(s, x) \in[0, T) \times \mathbb{R}^{d}$ that

$$
\begin{aligned}
& \mathbf{U}_{k, \rho}^{\theta}(s, x)=(g(x), 0)+\sum_{i=1}^{\mathfrak{m}_{k, \rho}} \frac{1}{\mathfrak{m}_{k, \rho}}\left[g\left(x+W_{T}^{(\theta, 0,-i)}-W_{s}^{(\theta, 0,-i)}\right)-g(x)\right]\left(1, \frac{W_{T}^{(\theta, 0,-i)}-W_{s}^{(\theta, 0,-i)}}{T-s}\right) \\
& \quad+\sum_{l=0}^{k-1} \sum_{i=1}^{m_{k-l, \rho}} \sum_{t \in(s, T]} \frac{q_{s}^{k-l, \rho}(t)}{m_{k-l, \rho}}\left[f\left(t, x+W_{t}^{(\theta, l, i)}-W_{s}^{(\theta, l, i)}, \mathbf{U}_{l, \rho}^{(\theta, l, i, t)}\left(t, x+W_{t}^{(\theta, l, i)}-W_{s}^{(\theta, l, i)}\right)\right)\right. \\
& \left.\quad-\mathbb{1}_{\mathbb{N}}(l) f\left(t, x+W_{t}^{(\theta, l, i)}-W_{s}^{(\theta, l, i)}, \mathbf{U}_{[l-1]^{+}, \rho}^{(\theta,-l, i, t}\left(t, x+W_{t}^{(\theta, l, i)}-W_{s}^{(\theta, l, i)}\right)\right)\right]\left(1, \frac{W_{t}^{(\theta, l, i)}-W_{s}^{(\theta, l, i)}}{t-s}\right) .
\end{aligned}
$$

We note that if $f$ does not depend on its last argument, then we only need the first components of the approximations and this simplifies all expressions; cf. (18). We also note that the term $g(x)$ cancels in the first components of the approximations.

\subsection{Numerical simulations of high-dimensional semilinear PDEs}

We applied the algorithm (12) to approximate the solutions at single space-time points of several semilinear PDEs from physics and financial mathematics such as

(i) a PDE arising from the recursive pricing model with default risk due to Duffie, Schroder, \& Skiadas [16],

(ii) a PDE arising from the valuation of derivative contracts with counterparty credit risk (see, e.g., Burgard \& Kjaer [10] and Henry-Labordère [27] for derivations of the PDE),

(iii) a PDE arising from pricing models for financial markets with different interest rates for borrowing and lending due to Bergman [7],

(iv) a version of the Allen-Cahn equation with a double well potential, and

(v) a PDE with an explicit solution whose three-dimensional version has been considered in Chassagneux [11].

We took $d=100$. All simulations are performed on a computer with a $2.8 \mathrm{GHz}$ Intel i7 processor and 16 GB RAM. We refer to [17] for the simulation results, MATLAB codes and further details concerning the numerical simulations. These results indicate that the proposed algorithm is efficient and practical for dealing with these high-dimensional PDEs.

\section{Convergence rate for the multilevel Picard iteration}

In this section we establish the convergence rate for semilinear heat equations in the case where the nonlinearity is independent of the gradient of the solution and satisfies the Lipschitztype condition (14) below and when the Gauß-Legendre formula (16) (see, e.g., [15] for more details) is used as the quadrature rule. More formally, we consider the special case of (2) where $\mu \equiv 0, \sigma \equiv \mathbb{1}_{d \times d}$ and $f$ does not depend on its last argument. 


\subsection{Setting}

Let $T, L \in(0, \infty), d \in \mathbb{N}, g \in C^{2}\left(\mathbb{R}^{d}, \mathbb{R}\right), \Theta=\cup_{n \in \mathbb{N}} \mathbb{R}^{n}$, let $\left(\Omega, \mathcal{F}, \mathbb{P},\left(\mathbb{F}_{t}\right)_{t \in[0, T]}\right)$ be a stochastic basis, let $W^{\theta}:[0, T] \times \Omega \rightarrow \mathbb{R}^{d}, \theta \in \Theta$, be independent standard $\left(\mathbb{F}_{t}\right)_{t \in[0, T]^{-}}$ Brownian motions with continuous sample paths, let $f:[0, T] \times \mathbb{R}^{d} \times \mathbb{R} \rightarrow \mathbb{R}$ be a Borel measurable function which satisfies for all $t \in[0, T], x \in \mathbb{R}^{d}, u_{1}, u_{2} \in \mathbb{R}$ that

$$
\left|f\left(t, x, u_{1}\right)-f\left(t, x, u_{2}\right)\right| \leq L\left|u_{1}-u_{2}\right|,
$$

let $F: \mathcal{M}\left(\mathcal{B}\left([0, T] \times \mathbb{R}^{d}\right), \mathcal{B}(\mathbb{R})\right) \rightarrow \mathcal{M}\left(\mathcal{B}\left([0, T] \times \mathbb{R}^{d}\right), \mathcal{B}(\mathbb{R})\right)$ be the function which satisfies for all $t \in[0, T], x \in \mathbb{R}^{d}, u \in \mathcal{M}\left(\mathcal{B}\left([0, T] \times \mathbb{R}^{d}\right), \mathcal{B}(\mathbb{R})\right)$ that $(F(u))(t, x)=$

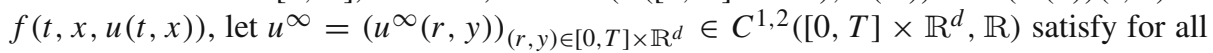
$r \in[0, T], y \in \mathbb{R}^{d}$ that $u^{\infty}(T, y)=g(y)$ and

$$
\partial_{r} u^{\infty}(r, y)+\frac{1}{2}\left(\Delta_{y} u^{\infty}\right)(r, y)+\left(F\left(u^{\infty}\right)\right)(r, y)=0,
$$

for every $n \in \mathbb{N}$ let $\left(c_{i}^{n}\right)_{i \in\{1, \ldots, n\}} \subseteq[-1,1]$ be the $n$ distinct roots of the Legendre polynomial $[-1,1] \ni x \mapsto \frac{1}{2^{n} n !} \frac{d^{n}}{d x^{n}}\left[\left(x^{2}-1\right)^{n}\right] \in \mathbb{R}$, for every $n \in \mathbb{N}$ let the function $q^{n,[-1,1]}:[-1,1] \rightarrow \mathbb{R}$ satisfy for all $t \in[-1,1]$ that

$$
q^{n,[-1,1]}(t)=\left[\mathbb{1}_{\left\{c_{1}^{n}, \ldots, c_{n}^{n}\right\}}(t)\right]\left[\int_{-1}^{1} \prod_{i \in\{1, \ldots, n\}, c_{i}^{n} \neq t} \frac{x-c_{i}^{n}}{t-c_{i}^{n}} d x\right],
$$

for every $n \in \mathbb{N}, a \in \mathbb{R}, b \in(a, \infty)$ let the function $q^{n,[a, a]}:[a, a] \rightarrow \mathbb{R}$ satisfy $q^{n,[a, a]}(a)=0$ and let the function $q^{n,[a, b]}:[a, b] \rightarrow \mathbb{R}$ satisfy for all $t \in[a, b]$ that $q^{n,[a, b]}(t)=\frac{b-a}{2} q^{n,[-1,1]}\left(\frac{2 t-(a+b)}{b-a}\right)$, let $\left(\bar{q}^{n, Q}\right)_{n, Q \in \mathbb{N}_{0}} \subseteq \mathcal{Q}_{T}$ satisfy for all $n, Q \in \mathbb{N}$, $t \in[0, T]$ that $\bar{q}^{0, Q}(t)=\mathbb{1}_{\{0\}}(t)$ and

$$
\bar{q}^{n, Q}(t)=\sum_{s \in[0, t]} \bar{q}^{n-1, Q}(s) q^{Q,[s, T]}(t),
$$

let $U_{n, M, Q}^{\theta}:[0, T] \times \mathbb{R}^{d} \times \Omega \rightarrow \mathbb{R}, n, M, Q \in \mathbb{Z}, \theta \in \Theta$, satisfy for all $n, M, Q \in \mathbb{N}$, $\theta \in \Theta,(t, x) \in[0, T] \times \mathbb{R}^{d}$ that $U_{0, M, Q}^{\theta}(t, x)=0$ and

$$
\begin{aligned}
& U_{n, M, Q}^{\theta}(t, x)=\frac{1}{M^{n}} \sum_{i=1}^{M^{n}} g\left(x+W_{T}^{(\theta, 0,-i)}-W_{t}^{(\theta, 0,-i)}\right) \\
& +\sum_{l=0}^{n-1} \sum_{i=1}^{M^{n-l}} \sum_{s \in[t, T]} \frac{q^{Q,[t, T]}(s)}{M^{n-l}}\left(F\left(U_{l, M, Q}^{(\theta, l, i, s)}\right)-\mathbb{1}_{\mathbb{N}}(l) F\left(U_{l-1, M, Q}^{(\theta,-l, i, s)}\right)\right)\left(s, x+W_{s}^{(\theta, l, i)}-W_{t}^{(\theta, l, i)}\right),
\end{aligned}
$$

and for every $n, Q \in \mathbb{N}_{0}$ let $\|\cdot\|_{n, Q}:\left(\mathcal{M}\left(\mathcal{B}\left(\mathbb{R}^{d}\right) \otimes \mathcal{F}, \mathcal{B}(\mathbb{R})\right)\right)^{[0, T]} \rightarrow[0, \infty]$ be the function which satisfies for all $V \in\left(\mathcal{M}\left(\mathcal{B}\left(\mathbb{R}^{d}\right) \otimes \mathcal{F}, \mathcal{B}(\mathbb{R})\right)\right)^{[0, T]}$ that

$$
\|V\|_{n, Q}=\sum_{t \in[0, T]} \bar{q}^{n, Q}(t)\left[\sup _{s \in[t, T]} \sup _{u \in[0, s]} \sup _{z \in \mathbb{R}^{d}} \sqrt{\mathbb{E}\left[\left|V\left(s, z+W_{u}^{0}\right)\right|^{2}\right]}\right] .
$$




\subsection{Pseudocode}

In this subsection a mathematical style pseudocode illustrates that the multilevel Picard approximations (18) can be easily implemented. We assume that the time horizon $T \in(0, \infty)$, the dimension $d \in \mathbb{N}$, the terminal condition $g: \mathbb{R}^{d} \rightarrow \mathbb{R}$, the (gradient-independent) nonlinearity $f:[0, T] \times \mathbb{R}^{d} \times \mathbb{R} \rightarrow \mathbb{R}$, the basis for the number of Monte Carlo samples $M \in \mathbb{N}$, the number of quadrature nodes $Q \in \mathbb{N}$, the increasingly ordered roots $c \in[-1,1]{ }^{Q}$ of the $Q$-th Legendre polynomial, and the corresponding Legendre quadrature weights $w \in$ $[0, \infty)^{Q}$ are global variables. For an implementation in MATLAB see [17].

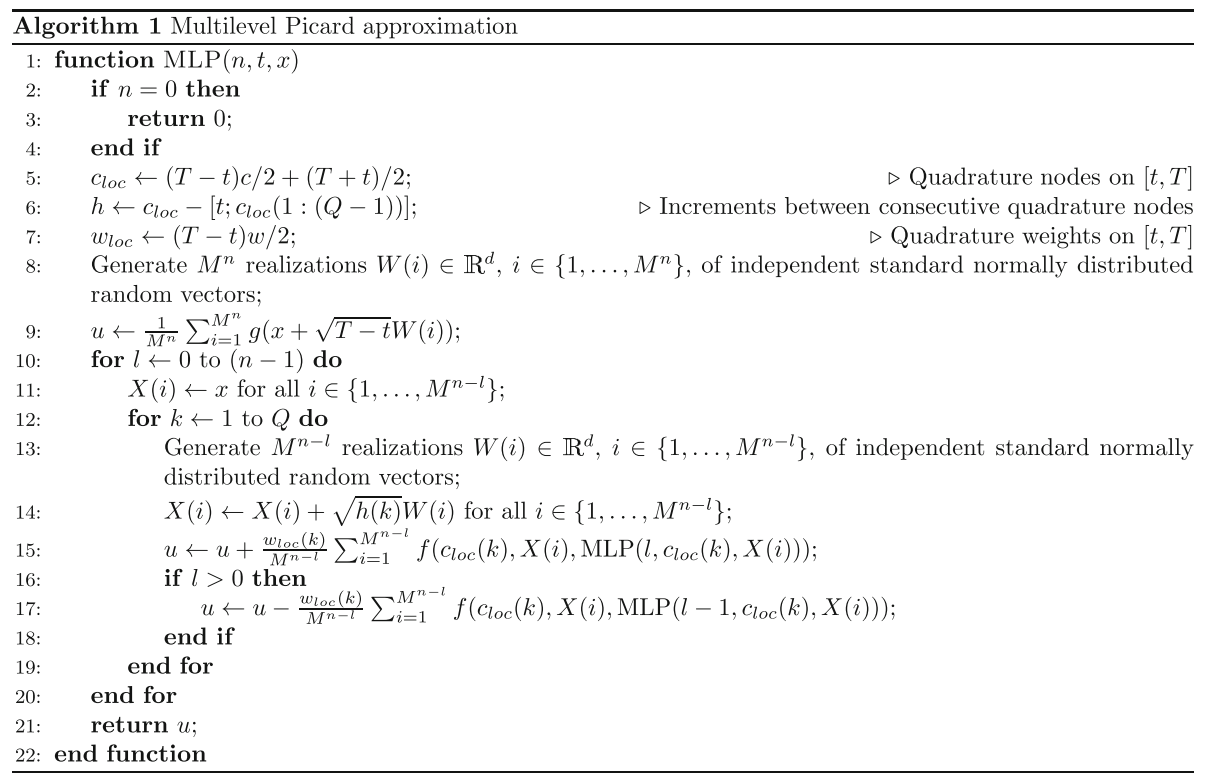

\subsection{Sketch of the proof}

Throughout this subsection assume the setting in Sect.3.1 and let $N, M, Q \in \mathbb{N}$. Theorem 3.12 provides an upper bound for the distance between the approximation $U_{N, M, Q}^{0}$ and the PDE solution $u^{\infty}$ measured in the seminorm $\|\cdot\|_{0, Q}$ given in (19). We establish this bound by splitting the global error $\left\|U_{N, M, Q}^{0}-u^{\infty}\right\|_{n, Q}$ into the Monte Carlo error $\left\|U_{N, M, Q}^{0}-\mathbb{E}\left[U_{N, M, Q}^{0}\right]\right\|_{n, Q}$ and the time discretization error $\left\|\mathbb{E}\left[U_{N, M, Q}^{0}\right]-u^{\infty}\right\|_{n, Q}$. To analyze the time discretization error, we employ the Feynman-Kac formula to obtain

$$
u^{\infty}(s, x)=\mathbb{E}\left[g\left(x+W_{T-s}^{0}\right)+\int_{s}^{T}\left(F\left(u^{\infty}\right)\right)\left(t, x+W_{t-s}^{0}\right) d t\right]
$$

for all $s \in[0, T], x \in \mathbb{R}^{d}$ (see Lemma 3.11 below). Moreover, the approximations admit the following Feynman-Kac-type representation

$$
\mathbb{E}\left[U_{N, M, Q}^{0}(s, x)\right]=\mathbb{E}\left[g\left(x+W_{T-s}^{0}\right)+\sum_{t \in[s, T]} q^{Q,[s, T]}(t)\left(F\left(U_{N-1, M, Q}^{0}\right)\right)\left(t, x+W_{t-s}^{0}\right)\right]
$$


for all $s \in[0, T], x \in \mathbb{R}^{d}$ (see Lemma3.10 below). This, (20) and the Lipschitz-type assumption (14) show that the time discretization error is bounded from above by the error of the $(N-1)$-th approximation $\left\|U_{N-1, M, Q}^{0}-u^{\infty}\right\|_{n+1, Q}$ and the error of the Gauß-Legendre quadrature rule applied to the function $[s, T] \ni t \mapsto \mathbb{E}\left[F\left(u^{\infty}\right)\left(t, x+W_{t-s}\right)\right] \in \mathbb{R}$ (see (52) below). Combining this with the established bound for the Monte Carlo error (see (50) below) results in the recursive inequality for the global error (53) that can be handled using a discrete Gronwall-type inequality. The error representation for Gauß-Legendre quadrature rules allows to further simplify the global error under suitable regularity assumptions (see Corollary 3.15 below). In Sect. 3.7 we provide upper bounds for the number of realizations of scalar standard normal random variables and for the number of function evaluations of $f$ and $g$ used to compute one realization of $U_{N, M, Q}^{0}(t, x)$ for a single point $(t, x) \in[0, T] \times \mathbb{R}^{d}$ in space-time. This and Corollary 3.15 prove in the case of the semilinear heat equation (15) that the computational complexity (see Corollary 3.18 for the precise definition hereof) of our proposed scheme grows linearly in the space dimension $d$ and polynomially in the inverse accuracy $\varepsilon^{-1}$ if the PDE solution $u^{\infty}$ is infinitely often differentiable and if the nonlinearity and the rescaled derivatives $\frac{\left(\frac{\partial}{\partial t}+\frac{1}{2} \Delta_{x}\right)^{k} u^{\infty}}{(k !)^{1-\alpha}}$ are uniformly bounded in time, space, $k \in \mathbb{N}_{0}$, and in the dimension for at least one $\alpha \in\left(0, \frac{1}{4}\right]$; see Corollary 3.18 below for details.

\subsection{Preliminary results for the Gauß-Legendre quadrature rules}

Lemma 3.1 (Gauß-Legendre over different intervals) Assume the setting in Sect.3.1, let $n \in$ $\mathbb{N}, s \in[0, T), t \in[0, s]$, and let $\psi:[0, T] \rightarrow[0, \infty]$ be a non-increasing function. Then we have

$$
\sum_{r \in[s, T]} q^{n,[s, T]}(r) \psi(r) \leq \sum_{r \in[t, T]} q^{n,[t, T]}(r) \psi(r) .
$$

Proof Observe that the fact that $t \leq s$ and the fact that $\forall i \in\{1, \ldots, n\}: c_{i}^{n} \in[-1,1]$ ensure that for all $i \in\{1, \ldots, n\}$ it holds that $\frac{T-s}{2} c_{i}^{n}+\frac{T+s}{2} \geq \frac{T-t}{2} c_{i}^{n}+\frac{T+t}{2}$. This and the fact that $\psi$ is non-increasing imply for all $i \in\{1, \ldots, n\}$ that $\psi\left(\frac{T-s}{2} c_{i}^{n}+\frac{T+s}{2}\right) \leq \psi\left(\frac{T-t}{2} c_{i}^{n}+\frac{T+t}{2}\right)$. Combining this with (16), the definitions of $q^{n,[s, T]}$ and $q^{n,[t, T]}$, and the fact that $T-s \leq T-t$ proves that

$$
\begin{aligned}
& \sum_{r \in[s, T]} q^{n,[s, T]}(r) \psi(r)=\sum_{i=1}^{n} q^{n,[s, T]}\left(\frac{T-s}{2} c_{i}^{n}+\frac{T+s}{2}\right) \psi\left(\frac{T-s}{2} c_{i}^{n}+\frac{T+s}{2}\right) \\
& =\sum_{i=1}^{n} \frac{T-s}{2} q^{n,[-1,1]}\left(c_{i}^{n}\right) \psi\left(\frac{T-s}{2} c_{i}^{n}+\frac{T+s}{2}\right) \leq \sum_{i=1}^{n} \frac{T-t}{2} q^{n,[-1,1]}\left(c_{i}^{n}\right) \psi\left(\frac{T-t}{2} c_{i}^{n}+\frac{T+t}{2}\right) \\
& =\sum_{i=1}^{n} q^{n,[t, T]}\left(\frac{T-t}{2} c_{i}^{n}+\frac{T+t}{2}\right) \psi\left(\frac{T-t}{2} c_{i}^{n}+\frac{T+t}{2}\right)=\sum_{r \in[t, T]} q^{n,[t, T]}(r) \psi(r) .
\end{aligned}
$$

This completes the proof of Lemma 3.1.

Lemma 3.2 Assume the setting in Sect.3.1 and let $Q \in \mathbb{N}$. Then for all $n \in \mathbb{N}_{0}, k \in$ $\mathbb{N}_{0} \cap[0,2 Q-n]$ we have

$$
\sum_{t \in[0, T]} \bar{q}^{n, Q}(t) \frac{(T-t)^{k}}{k !}=\frac{T^{n+k}}{(n+k) !} .
$$


Proof First, note that the fact that the Gauß-Legendre quadrature rule $C([0, T], \mathbb{R}) \ni \varphi \mapsto$ $\sum_{t \in[0, T]} q^{Q,[0, T]}(t) \varphi(t) \in \mathbb{R}$ integrates polynomials of order less than $2 Q$ exactly implies that for all $s \in[0, T], k \in \mathbb{N}_{0} \cap[0,2 Q)$ it holds that

$$
\sum_{t \in[s, T]} q^{Q,[s, T]}(t) \frac{(T-t)^{k}}{k !}=\int_{s}^{T} \frac{(T-t)^{k}}{k !} d t=\frac{(T-s)^{k+1}}{(k+1) !} .
$$

We now prove (24) by induction on $n \in \mathbb{N}_{0}$. For the base case $n=0$ we note that for all $k \in \mathbb{N}_{0}$ it holds that

$$
\sum_{t \in[0, T]} \bar{q}^{0, Q}(t) \frac{(T-t)^{k}}{k !}=\sum_{t \in[0, T]} \mathbb{1}_{\{0\}}(t) \frac{(T-t)^{k}}{k !}=\frac{T^{k}}{k !} .
$$

This establishes (24) in the base case $n=0$. For the induction step $\mathbb{N}_{0} \ni n \rightarrow n+1 \in \mathbb{N}$ we observe that (25) and the induction hypothesis imply that for all $k \in \mathbb{N}_{0} \cap[0,2 Q-n-1]$ it holds that

$$
\begin{aligned}
\sum_{t \in[0, T]} \bar{q}^{\left.n+1, Q_{(}\right) \frac{(T-t)^{k}}{k !}} & =\sum_{t \in[0, T]}\left[\sum_{s \in[0, t]} \bar{q}^{n, Q}(s) q^{Q,[s, T]}(t)\right] \frac{(T-t)^{k}}{k !} \\
& =\sum_{s \in[0, T]} \bar{q}^{n, Q}(s)\left[\sum_{t \in[s, T]} q^{Q,[s, T]}(t) \frac{(T-t)^{k}}{k !}\right] \\
& =\sum_{s \in[0, T]} \bar{q}^{n, Q}(s) \frac{(T-s)^{k+1}}{(k+1) !}=\frac{T^{n+1+k}}{(n+1+k) !} .
\end{aligned}
$$

This finishes the induction step $\mathbb{N}_{0} \ni n \rightarrow n+1 \in \mathbb{N}$. Induction hence establishes (24). The proof of Lemma 3.2 is thus completed.

\subsection{Preliminary results for the seminorms}

We refer to a $[0, \infty]$-valued function as seminorm if it is subadditive and absolutely homogeneous. In particular, we do not require seminorms to have finite values. The proof of the following lemma is clear and therefore omitted.

Lemma 3.3 (Seminorm property) Assume the setting in Sect.3.1 and let $k \in \mathbb{N}_{0}, Q \in \mathbb{N}$. Then the function $\left(\mathcal{M}\left(\mathcal{B}\left(\mathbb{R}^{d}\right) \otimes \mathcal{F}, \mathcal{B}(\mathbb{R})\right)\right)^{[0, T]} \ni U \mapsto\|U\|_{k, Q} \in[0, \infty]$ is a seminorm in the sense that it is subadditive, nonnegative, and absolutely homogeneous.

The following lemma implies that Monte Carlo averages converge in our seminorms with rate $1 / 2$.

Lemma 3.4 (Linear combinations of i.i.d. random variables) Assume the setting in Sect.3.1, let $k \in \mathbb{N}_{0}, n, Q \in \mathbb{N}, r_{1}, \ldots, r_{n} \in \mathbb{R}, V_{1}, \ldots, V_{n} \in\left(\mathcal{M}\left(\mathcal{B}\left(\mathbb{R}^{d}\right) \otimes \mathcal{F}, \mathcal{B}(\mathbb{R})\right)\right)^{[0, T]}$ satisfy for all $s \in[0, T], x \in \mathbb{R}^{d}$ that $V_{1}(s, x), \ldots, V_{n}(s, x)$ are integrable i.i.d. random variables, and assume for all $s \in[0, T]$ that $V_{1}(s, \cdot), \ldots, V_{n}(s, \cdot), W^{0}$ are independent. Then

$$
\left\|\sum_{i=1}^{n} r_{i}\left(V_{i}-\mathbb{E}\left[V_{i}\right]\right)\right\|_{k, Q}=\left\|\left(V_{1}-\mathbb{E}\left[V_{1}\right]\right)\right\|_{k, Q} \sqrt{\sum_{i=1}^{n}\left|r_{i}\right|^{2}} \leq\left\|V_{1}\right\|_{k, Q} \sqrt{\sum_{i=1}^{n}\left|r_{i}\right|^{2}} .
$$


Proof The definition(19) of the seminorm, the disintegration theorem, the fact that for all $s \in[0, T], x \in \mathbb{R}^{d}$ it holds that $V_{1}(s, \cdot), \ldots, V_{n}(s, \cdot), W^{0}$ are independent, and the fact that for all $s \in[0, T], x \in \mathbb{R}^{d}$ it holds that $V_{1}(s, x), \ldots, V_{n}(s, x)$ are i.i.d. imply that

$$
\begin{aligned}
& \left\|\sum_{i=1}^{n} r_{i}\left(V_{i}-\mathbb{E}\left[V_{i}\right]\right)\right\|_{k, Q} \\
& =\sum_{t \in[0, T]} \bar{q}^{k, Q}(t)\left[\operatorname { s u p } _ { s \in [ t , T ] } \operatorname { s u p } _ { u \in [ 0 , s ] } \operatorname { s u p } _ { z \in \mathbb { R } ^ { d } } \mathbb { E } \left[\mathbb { E } \left[\mid \sum_{i=1}^{n} r_{i}\left(V_{i}\left(s, z+W_{u}^{0}\right)\right.\right.\right.\right. \\
& \left.\left.\left.\left.-\mathbb{E}\left[V_{i}\left(s, z+W_{u}^{0}\right) \mid W^{0}\right]\right)\left.\right|^{2} \mid W^{0}\right]\right]\right]^{\frac{1}{2}} \\
& =\sum_{t \in[0, T]} \bar{q}^{k, Q}(t)\left[\sup _{s \in[t, T]} \sup _{u \in[0, s]} \sup _{z \in \mathbb{R}^{d}} \mathbb{E}\left[\operatorname{Var}\left(\sum_{i=1}^{n} r_{i} V_{i}\left(s, z+W_{u}^{0}\right) \mid W^{0}\right)\right]\right]^{\frac{1}{2}} \\
& =\sum_{t \in[0, T]} \bar{q}^{k, Q}(t)\left[\sup _{s \in[t, T]} \sup _{u \in[0, s]} \sup _{z \in \mathbb{R}^{d}} \mathbb{E}\left[\sum_{i=1}^{n}\left|r_{i}\right|^{2} \operatorname{Var}\left(V_{1}\left(s, z+W_{u}^{0}\right) \mid W^{0}\right)\right]\right]^{\frac{1}{2}} \\
& =\left\|V_{1}-\mathbb{E}\left[V_{1}\right]\right\|_{k, Q} \sqrt{\sum_{i=1}^{n}\left|r_{i}\right|^{2}} \\
& \leq \sum_{t \in[0, T]} \bar{q}^{k, Q_{(t)}}\left[\sup _{s \in[t, T]} \sup _{u \in[0, s]} \sup _{z \in \mathbb{R}^{d}} \mathbb{E}\left[\mathbb{E}\left[\left|V_{1}\left(s, z+W_{u}^{0}\right)\right|^{2} \mid W^{0}\right]\right]\right]^{\frac{1}{2}} \sqrt{\sum_{i=1}^{n}\left|r_{i}\right|^{2}} \\
& =\left\|V_{1}\right\|_{k, Q} \sqrt{\sum_{i=1}^{n}\left|r_{i}\right|^{2}} \text {. }
\end{aligned}
$$

This completes the proof of Lemma 3.4.

Lemma 3.5 (Lipschitz property) Assume the setting in Sect.3.1, let $k \in \mathbb{N}_{0}, Q \in \mathbb{N}$, and let $U, V \in\left(\mathcal{M}\left(\mathcal{B}\left(\mathbb{R}^{d}\right) \otimes \mathcal{F}, \mathcal{B}(\mathbb{R})\right)\right)^{[0, T]}$. Then

$$
\|F(U)-F(V)\|_{k, Q} \leq L\|U-V\|_{k, Q} .
$$

Proof The definition (19) of the seminorm and the global Lipschitz property (14) of $F$ imply that

$$
\begin{aligned}
& \|F(U)-F(V)\|_{k, Q} \\
& =\sum_{t \in[0, T]} \bar{q}^{k, Q}(t)\left[\sup _{s \in[t, T]} \sup _{u \in[0, s]} \sup _{z \in \mathbb{R}^{d}} \mathbb{E}\left[\left|(F(U))\left(s, z+W_{u}^{0}\right)-(F(V))\left(s, z+W_{u}^{0}\right)\right|^{2}\right]\right]^{\frac{1}{2}} \\
& \leq \sum_{t \in[0, T]} \bar{q}^{k, Q}(t)\left[\sup _{s \in[t, T]} \sup _{u \in[0, s]} \sup _{z \in \mathbb{R}^{d}} L^{2} \mathbb{E}\left[\left|U\left(s, z+W_{u}^{0}\right)-V\left(s, z+W_{u}^{0}\right)\right|^{2}\right]\right]^{\frac{1}{2}} \\
& =L\|U-V\|_{k, Q} .
\end{aligned}
$$

This completes the proof of Lemma 3.5 
Lemma 3.6 Assume the setting in Sect.3.1, let $k \in \mathbb{N}_{0}, Q \in \mathbb{N}$, and let $U:[0, T] \rightarrow$ $\mathcal{M}\left(\mathcal{B}\left(\mathbb{R}^{d}\right) \otimes \mathcal{F}, \mathcal{B}(\mathbb{R})\right)$ satisfy for all $s \in[0, T]$ that $U(s, \cdot)$ and $W^{0}$ are independent. Then

$$
\left\|[0, T] \times \mathbb{R}^{d} \ni(s, z) \mapsto \sum_{t \in[s, T]} q^{Q,[s, T]}(t) U\left(t, z+W_{t}^{0}-W_{s}^{0}\right) \in \mathbb{R}\right\|_{k, Q} \leq\|U\|_{k+1, Q} .
$$

Proof The definition(19) of the seminorm, the triangle inequality, independence, the disintegration theorem, Lemma 3.1, and the definition (17) of $\bar{q}^{k+1, Q}$ yield that

$$
\begin{aligned}
\left\|[0, T] \times \mathbb{R}^{d} \ni(s, z) \mapsto \sum_{r \in[s, T]} q^{Q,[s, T]}(r) U\left(r, z+W_{r}^{0}-W_{s}^{0}\right) \in \mathbb{R}\right\|_{k, Q} \\
=\sum_{t \in[0, T]} \bar{q}^{k, Q}(t)\left[\sup _{s \in[t, T]} \sup _{u \in[0, s]} \sup _{z \in \mathbb{R}^{d}}\left(\mathbb{E}\left[\left|\sum_{r \in[s, T]} q^{Q,[s, T]}(r) U\left(r, z+W_{u}^{0}+W_{r}^{0}-W_{s}^{0}\right)\right|^{2}\right]\right)^{\frac{1}{2}}\right] \\
\leq \sum_{t \in[0, T]} \bar{q}^{k, Q}(t)\left[\sup _{s \in[t, T]} \sup _{u \in[0, s]} \sup _{z \in \mathbb{R}^{d}} \sum_{r \in[s, T]} q^{Q,[s, T]}(r)\left(\mathbb{E}\left[\left|U\left(r, z+W_{u}^{0}+W_{r}^{0}-W_{s}^{0}\right)\right|^{2}\right]\right)^{\frac{1}{2}}\right] \\
\leq \sum_{t \in[0, T]} \bar{q}^{k, Q}(t) \sup _{s \in[t, T]} \sum_{r \in[s, T]} q^{Q,[s, T]}(r)\left[\sup _{v \in[r, T]} \sup _{u \in[0, v]} \sup _{z \in \mathbb{R}^{d}} \mathbb{E}\left[\left|U\left(v, z+W_{u}^{0}\right)\right|^{2}\right]\right]^{\frac{1}{2}} \\
=\sum_{t \in[0, T]} \bar{q}^{k, Q}(t) \sum_{r \in[t, T]} q_{q,[t, T]}[r)\left[\sup _{v \in[r, T]} \sup _{u \in[0, v]} \sup _{z \in \mathbb{R}^{d}} \mathbb{E}\left[\left|U\left(v, z+W_{u}^{0}\right)\right|^{2}\right]\right]^{\frac{1}{2}} \\
=\sum_{r \in[0, T]} \bar{q}^{k+1, Q}(r)\left[\sup _{v \in[r, T]} \sup _{u \in[0, v]} \sup _{z \in \mathbb{R}^{d}} \mathbb{E}\left[\left|U\left(v, z+W_{u}^{0}\right)\right|^{2}\right]\right]^{\frac{1}{2}} \\
=\|U\|_{k+1, Q} .
\end{aligned}
$$

This completes the proof of Lemma 3.6.

Lemma 3.7 (Expectation) Assume the setting in Sect.3.1, let $k \in \mathbb{N}_{0}, Q \in \mathbb{N}$, and let $U:[0, T] \rightarrow \mathcal{M}\left(\mathcal{B}\left(\mathbb{R}^{d}\right) \otimes \mathcal{F}, \mathcal{B}(\mathbb{R})\right)$ satisfy for all $(s, x) \in[0, T] \times \mathbb{R}^{d}$ that $\mathbb{E}[|U(s, x)|]<$ $\infty$ and that $U(s, \cdot)$ and $\left(W_{t}^{0}\right)_{t \in[0, s]}$ are independent. Then

$$
\|\mathbb{E}[U]\|_{k, Q} \leq\|U\|_{k, Q}
$$

Proof The definition(19) of the seminorm, Jensen's inequality, independence, and the disintegration theorem imply that

$$
\begin{aligned}
\|\mathbb{E}[U]\|_{k, Q} & =\sum_{t \in[0, T]} \bar{q}^{k, Q_{(t)}}\left[\sup _{s \in[t, T]} \sup _{u \in[0, s]} \sup _{z \in \mathbb{R}^{d}}\left(\int|\mathbb{E}[U(s, y)]|^{2} \mathbb{P}\left(z+W_{u}^{0} \in d y\right)\right)^{\frac{1}{2}}\right] \\
& \leq \sum_{t \in[0, T]} \bar{q}^{k, Q_{(t)}}\left[\sup _{s \in[t, T]} \sup _{u \in[0, s]} \sup _{z \in \mathbb{R}^{d}}\left(\int \mathbb{E}\left[|U(s, y)|^{2}\right] \mathbb{P}\left(z+W_{u}^{0} \in d y\right)\right)^{\frac{1}{2}}\right]
\end{aligned}
$$




$$
=\sum_{t \in[0, T]} \bar{q}^{k, Q}(t)\left[\sup _{s \in[t, T]} \sup _{u \in[0, s]} \sup _{z \in \mathbb{R}^{d}}\left(\mathbb{E}\left[\left|U\left(s, z+W_{u}^{0}\right)\right|^{2}\right]\right)^{\frac{1}{2}}\right]=\|U\|_{k, Q} .
$$

This completes the proof of Lemma 3.7.

The following lemma specifies the values of our seminorms of constant functions. It follows directly from the definition(19) of the seminorms and from Lemma3.2. Its proof is therefore omitted.

Lemma 3.8 (Seminorm of constants) Assume the setting in Sect.3.1 and let $Q \in \mathbb{N}, k \in$ $\mathbb{N}_{0} \cap[0,2 Q]$. Then $\|1\|_{k, Q}=\frac{T^{k}}{k !}$.

\subsection{Error analysis for the multilevel Picard iteration}

Lemma 3.9 (Distributional and independence properties of approximations) Assume the setting in Sect.3.1. Then

(i) it holds for all $n \in \mathbb{N}_{0}, M, Q \in \mathbb{N}, \theta \in \Theta$ that $U_{n, M, Q}^{\theta} \in\left(\mathcal{M}\left(\mathcal{B}\left(\mathbb{R}^{d}\right) \otimes \mathcal{F}, \mathcal{B}(\mathbb{R})\right)\right)^{[0, T]}$,

(ii) it holds for all $n \in \mathbb{N}_{0}, M, Q \in \mathbb{N}, \theta \in \Theta, t \in[0, T]$ that $\sigma\left(U_{n, M, Q}^{\theta}(t, \cdot)\right) \subseteq$ $\sigma\left(\left(W^{(\theta, \vartheta)}\right) \vartheta \in \Theta\right)$,

(iii) it holds for all $n \in \mathbb{N}_{0}, m, M, Q \in \mathbb{N}, \theta \in \mathbb{R}^{m}, t \in[0, T]$ that $U_{n, M, Q}^{\theta}(t, \cdot)$ and $\left(W^{\vartheta}\right)_{\vartheta \in \cup_{k=1}^{m}} \mathbb{R}^{k}$ are independent,

(iv) it holds for all $n \in \mathbb{N}_{0}, m, M, Q \in \mathbb{N}, t \in[0, T], \theta, \vartheta \in \mathbb{R}^{m}$ with $\theta \neq \vartheta$ that $U_{n, M, Q}^{\theta}(t, \cdot)$ and $U_{n, M, Q}^{\vartheta}(t, \cdot)$ are independent, and

(v) it holds for all $n \in \mathbb{N}_{0}, M, Q \in \mathbb{N}, t \in[0, T], x \in \mathbb{R}^{d}$ that $\left(U_{n, M, Q}^{\theta}(t, x)\right)_{\theta \in \Theta}$ are identically distributed.

Proof of Lemma 3.9 First note that the fact that for all $s, t \in[0, T], \theta \in \Theta$ it holds that $\mathbb{R}^{d} \times$ $\Omega \ni(x, \omega) \mapsto x+W_{s}^{\theta}(\omega)-W_{t}^{\theta}(\omega) \in \mathbb{R}^{d}$ is $\left(\mathcal{B}\left(\mathbb{R}^{d}\right) \otimes \mathcal{F}\right) / \mathcal{B}\left(\mathbb{R}^{d}\right)$ measurable, the fact that for all $s \in[0, T]$ it holds that $\mathbb{R}^{d} \times \mathbb{R} \ni(x, u) \mapsto f(s, x, u) \in \mathbb{R}$ is $\left(\mathcal{B}\left(\mathbb{R}^{d}\right) \otimes \mathcal{B}(\mathbb{R})\right) / \mathcal{B}(\mathbb{R})$ measurable, the fact that $g \in C^{2}\left(\mathbb{R}^{d}, \mathbb{R}\right)$, the fact that for all $M, Q \in \mathbb{N}, \theta \in \Theta, t \in[0, T]$, $x \in \mathbb{R}^{d}$ it holds that $U_{0, M, Q}^{\theta}(t, x)=0$, and (18) prove item(i) and item(ii). Furthermore, observe that item (ii) and the fact that for all $m \in \mathbb{N}, \theta \in \mathbb{R}^{m}$ it holds that $\left(W^{(\theta, \vartheta)}\right)_{\vartheta \in \Theta}$ and $\left(W^{\vartheta}\right)_{\vartheta \in \cup_{k=1}^{m}} \mathbb{R}^{k}$ are independent establish item (iii). Moreover, note that item (ii) and the fact that for all $m \in \mathbb{N}, \theta, \vartheta \in \mathbb{R}^{m}$ with $\theta \neq \vartheta$ it holds that $\left(W^{(\theta, \gamma)}\right)_{\gamma \in \Theta}$ and $\left(W^{(\vartheta, \gamma)}\right)_{\gamma \in \Theta}$ are independent implies item(iv). Finally, observe that the fact that for all $M, Q \in \mathbb{N}, \theta \in \Theta$, $t \in[0, T], x \in \mathbb{R}^{d}$ it holds that $U_{0, M, Q}^{\theta}(t, x)=0$, the fact that $\left(W^{\theta}\right)_{\theta \in \Theta}$ are identically distributed, and items (i)-(iv) establish item(v). The proof of Lemma3.9 is thus completed.

Lemma 3.10 (Approximations are integrable) Assume the setting in Subsection 3.1, let $z \in$ $\mathbb{R}^{d}, M, Q \in \mathbb{N}$, and assume for all $s \in[0, T], t \in[s, T]$ that $\mathbb{E}\left[\left|g\left(z+W_{t}^{0}\right)\right|+\mid(F(0))(t, z+\right.$ $\left.\left.W_{s}^{0}\right) \mid\right]<\infty$. Then

(i) for all $n \in \mathbb{N}_{0}, \theta \in \Theta, s \in[0, T], t \in[s, T]$ it holds that

$$
\mathbb{E}\left[\left|U_{n, M, Q}^{\theta}\left(t, z+W_{s}^{0}\right)\right|+\left|\left(F\left(U_{n, M, Q}^{\theta}\right)\right)\left(t, z+W_{s}^{0}\right)\right|\right]<\infty
$$

and 
(ii) for all $n \in \mathbb{N}_{0}, \theta \in \Theta, s \in[0, T]$ it holds that

$$
\mathbb{E}\left[U_{n+1, M, Q}^{\theta}(s, z)\right]=\mathbb{E}\left[g\left(z+W_{T-s}^{0}\right)+\sum_{t \in[s, T]} q^{Q,[s, T]}(t)\left(F\left(U_{n, M, Q}^{\theta}\right)\right)\left(t, z+W_{t-s}^{0}\right)\right] .
$$

Proof We prove item(i) by induction on $n \in \mathbb{N}_{0}$. For the base case $n=0$ we note that for all $\theta \in \Theta, s \in[0, T], t \in[s, T]$ it holds that

$$
\mathbb{E}\left[\left|U_{0, M, Q}^{\theta}\left(t, z+W_{s}^{0}\right)\right|+\left|\left(F\left(U_{0, M, Q}^{\theta}\right)\right)\left(t, z+W_{s}^{0}\right)\right|\right]=\mathbb{E}\left[\left|(F(0))\left(t, z+W_{s}^{0}\right)\right|\right]<\infty .
$$

This establishes item (i) in the base case $n=0$. For the induction step $\mathbb{N}_{0} \ni n \rightarrow n+1 \in \mathbb{N}$ let $n \in \mathbb{N}_{0}$ and assume that item(i) holds for $n=0, n=1, \ldots, n=n$. The induction hypothesis, (18) and Lemma 3.9 imply that for all $\theta \in \Theta, s \in[0, T], t \in[s, T]$ it holds that

$$
\begin{aligned}
& \mathbb{E}\left[\left|U_{n+1, M, Q}^{\theta}\left(t, z+W_{s}^{0}\right)\right|\right] \leq \mathbb{E}\left[\left|g\left(z+W_{T-t+s}^{0}\right)\right|\right] \\
& +\sum_{l=0}^{n} \sum_{r \in[t, T]} \frac{q^{Q,[t, T]}(r)}{M^{n+1-l}} \sum_{i=1}^{M^{n+1-l}} \sum_{k \in\{l-1, l\} \cap \mathbb{N}_{0}} \max _{j \in\{-l, l\}} \mathbb{E}\left[\left|\left(F\left(U_{k, M, Q}^{(\theta, j, i, r)}\right)\right)\left(r, z+W_{s+r-t}^{0}\right)\right|\right]<\infty .
\end{aligned}
$$

Combining this with (14) proves for all $\theta \in \Theta, s \in[0, T], t \in[s, T]$ that

$$
\begin{aligned}
& \mathbb{E}\left[\mid\left(F\left(U_{n+1, M, Q}^{\theta}\right)\left(t, z+W_{s}^{0}\right) \mid\right]\right. \\
& \quad \leq \mathbb{E}\left[\left|\left(F\left(U_{n+1, M, Q}^{\theta}\right)\right)\left(t, z+W_{s}^{0}\right)-(F(0))\left(t, z+W_{s}^{0}\right)\right|\right]+\mathbb{E}\left[\left|(F(0))\left(t, z+W_{s}^{0}\right)\right|\right] \\
& \quad \leq L \mathbb{E}\left[\left|U_{n+1, M, Q}^{\theta}\left(t, z+W_{s}^{0}\right)\right|\right]+\mathbb{E}\left[\left|(F(0))\left(t, z+W_{s}^{0}\right)\right|\right]<\infty .
\end{aligned}
$$

This finishes the induction step $\mathbb{N}_{0} \ni n \rightarrow n+1 \in \mathbb{N}$. Induction hence establishes item(i). Next we note that (18), Lemma3.9, and a telescope argument yield that for all $n \in \mathbb{N}_{0}$, $\theta \in \Theta, s \in[0, T]$ it holds that

$$
\begin{aligned}
\mathbb{E} & {\left[U_{n+1, M, Q}^{\theta}(s, z)\right]-\mathbb{E}\left[g\left(z+W_{T-s}^{0}\right)\right] } \\
& =\sum_{l=0}^{n} \sum_{v \in[s, T]} q^{Q,[s, T]}(v) \mathbb{E}\left[\left(F\left(U_{l, M, Q}^{0}\right)-\mathbb{1}_{\mathbb{N}}(l) F\left(U_{l-1, M, Q}^{0}\right)\left(v, z+W_{v}^{0}-W_{s}^{0}\right)\right]\right. \\
& =\sum_{v \in[s, T]} q^{Q,[s, T]}(v) \mathbb{E}\left[\left(F\left(U_{n, M, Q}^{0}\right)\right)\left(v, z+W_{v}^{0}-W_{s}^{0}\right)\right] \\
& =\mathbb{E}\left[\sum_{v \in[s, T]} q^{Q,[s, T]}(v)\left(F\left(U_{n, M, Q}^{\theta}\right)\right)\left(v, z+W_{v-s}^{0}\right)\right]
\end{aligned}
$$

This establishes item (ii). The proof of Lemma 3.10 is thus completed.

The following nonlinear Feynman-Kac formula is essentially known in the literature (cf., e.g., [36, Theorem 5.7.6]) under different assumptions. 
Lemma 3.11 (Nonlinear Feynman-Kac formula) Assume the setting in Sect.3.1, let $z \in \mathbb{R}^{d}$, and assume for all $s \in[0, T]$ that

$$
\mathbb{E}\left[\sup _{t \in[s, T]}\left|u^{\infty}\left(t, z+W_{t-s}^{0}\right)\right|+\int_{s}^{T}\left|(F(0))\left(t, z+W_{t-s}^{0}\right)\right| d t\right]<\infty .
$$

Then

(i) for all $s \in[0, T]$ it holds that

$$
\mathbb{E}\left[\sup _{t \in[s, T]}\left|u^{\infty}\left(t, z+W_{t}^{0}-W_{s}^{0}\right)\right|+\int_{s}^{T}\left|\left(F\left(u^{\infty}\right)\right)\left(t, z+W_{t}^{0}-W_{s}^{0}\right)\right| d t\right]<\infty
$$

and

(ii) for all $s \in[0, T]$ it holds that

$$
u^{\infty}(s, z)-\mathbb{E}\left[g\left(z+W_{T-s}^{0}\right)\right]=\mathbb{E}\left[\int_{s}^{T}\left(F\left(u^{\infty}\right)\right)\left(t, z+W_{t-s}^{0}\right) d t\right] .
$$

Proof Note that (14) and (42) imply item(i). Next Itô's formula and the PDE(15) ensure that for all $s \in[0, T], t \in[s, T]$ it holds $\mathbb{P}$-a.s. that

$$
\begin{aligned}
& u^{\infty}\left(t, z+W_{t}^{0}-W_{s}^{0}\right)-u^{\infty}(s, z) \\
& =\int_{s}^{t}\left(\frac{\partial}{\partial r} u^{\infty}+\frac{1}{2} \Delta_{y} u^{\infty}\right)\left(r, z+W_{r}^{0}-W_{s}^{0}\right) d r+\int_{s}^{t}\left\langle\left(\nabla_{y} u^{\infty}\right)\left(r, z+W_{r}^{0}-W_{s}^{0}\right), d W_{r}^{0}\right\rangle \\
& =-\int_{s}^{t}\left(F\left(u^{\infty}\right)\right)\left(r, z+W_{r}^{0}-W_{s}^{0}\right) d r+\int_{s}^{t}\left\langle\left(\nabla_{y} u^{\infty}\right)\left(r, z+W_{r}^{0}-W_{s}^{0}\right), d W_{r}^{0}\right\rangle .
\end{aligned}
$$

This and (43) show that for all $s \in[0, T]$ it holds that $\mathbb{E}\left[\sup _{t \in[s, T]} \mid \int_{s}^{t}\left\langle\left(\nabla_{y} u^{\infty}\right)\left(r, z+W_{r}^{0}-\right.\right.\right.$ $\left.\left.\left.W_{s}^{0}\right), d W_{r}^{0}\right\rangle \mid\right]<\infty$. This and the dominated convergence theorem ensure for all $s \in[0, T]$ that $\mathbb{E}\left[\int_{s}^{T}\left\langle\left(\nabla_{y} u^{\infty}\right)\left(t, z+W_{t}^{0}-W_{s}^{0}\right), d W_{t}^{0}\right\rangle\right]=0$. This and (45) prove for all $s \in[0, T]$ that

$$
\begin{aligned}
& u^{\infty}(s, z)-\mathbb{E}\left[g\left(z+W_{T-s}^{0}\right)\right]=u^{\infty}(s, z)-\mathbb{E}\left[u^{\infty}\left(T, z+W_{T}^{0}-W_{s}^{0}\right)\right] \\
& \quad=\mathbb{E}\left[\int_{s}^{T}\left(F\left(u^{\infty}\right)\right)\left(t, z+W_{t-s}^{0}\right) d t\right] .
\end{aligned}
$$

This finishes the proof of Lemma 3.11.

Theorem 3.12 Assume the setting in Sect.3.1, let $M, Q \in \mathbb{N}, N \in\{1, \ldots, 2 Q-1\}, \theta \in \Theta$, and assume for all $z \in \mathbb{R}^{d}, s \in[0, T]$ that

$$
\mathbb{E}\left[\sup _{t \in[s, T]}\left|u^{\infty}\left(t, z+W_{t-s}^{0}\right)\right|\right]+\sup _{t \in[s, T]} \mathbb{E}\left[\left|(F(0))\left(t, z+W_{t-s}^{0}\right)\right|\right]<\infty .
$$

Then we have

$$
\begin{aligned}
& \left\|U_{N, M, Q}^{\theta}-u^{\infty}\right\|_{0, Q} \leq(1+2 L)^{N-1}\left\{L \sup _{i \in\{1,2, \ldots, N\}} \frac{\left\|u^{\infty}\right\|_{i, Q}}{\sqrt{M^{N-i}}}\right. \\
& +\left[\sup _{i \in\{0,1, \ldots, N-1\}} \frac{T^{i}}{i ! \sqrt{M^{N-i}}}\right]\left[\sup _{z \in \mathbb{R}^{d}} \sup _{s \in[0, T]}\left\|g\left(z+W_{s}^{0}\right)\right\|_{L^{2}(\mathbb{P} ; \mathbb{R})}\right.
\end{aligned}
$$




$$
\begin{aligned}
& \left.+T \sup _{z \in \mathbb{R}^{d} r, s \in[0, T]} \sup _{\substack{t \in[0, T], r \in[0, t], z \in \mathbb{R}^{d}}}\left\|(F(0))\left(r, z+W_{s}^{0}\right)\right\|_{L^{2}(\mathbb{P} ; \mathbb{R})}\right] \\
& +e^{T} \sup _{s \in[t, T]} q^{Q,[t, T]}(s)\left(F\left(u^{\infty}\right)\right)\left(s, z+W_{r+s-t}^{0}\right) \\
& \left.\left.-\int_{t}^{T}\left(F\left(u^{\infty}\right)\right)\left(s, z+W_{r+s-t}^{0}\right) d s \mid W_{r}^{0}\right] \|_{L^{2}(\mathbb{P} ; \mathbb{R})}\right\}
\end{aligned}
$$

Proof Throughout this proof assume w.l.o.g. that the right-hand side of (48) is finite, assume w.l.o.g. that $\theta=0$ (the case $\theta \neq 0$ follows from the case $\theta=0$ ), let $\varepsilon \in[0, \infty)$ be the real number given by (cf. item(i) of Lemma 3.11)

$$
\begin{aligned}
\varepsilon= & \sup _{\substack{t \in[0, T], z \in \mathbb{R}^{d}}} \sup _{u \in[0, t]} \| \mathbb{E}\left[\sum_{s \in[t, T]} q^{Q,[t, T]}(s)\left(F\left(u^{\infty}\right)\right)\left(s, z+W_{u+s-t}^{0}\right)\right. \\
& \left.-\int_{t}^{T}\left(F\left(u^{\infty}\right)\right)\left(s, z+W_{u+s-t}^{0}\right) d s \mid W_{u}^{0}\right] \|_{L^{2}(\mathbb{P} ; \mathbb{R})},
\end{aligned}
$$

and let $\left(e_{n}\right)_{n \in\{0,1, \ldots, N\}} \subseteq[0, \infty]$ be the extended real numbers which satisfy for all $n \in$ $\{0,1, \ldots, N\}$ that

$$
e_{n}=\sup \left\{\sqrt{M^{-j}}\left\|U_{n, M, Q}^{0}-u^{\infty}\right\|_{k, Q}: k, j \in \mathbb{N}_{0}, k+j+n=N\right\} .
$$

First, we analyze the Monte Carlo error. Item(i) of Lemma 3.10 shows for all $n \in \mathbb{N}_{0}$, $(t, z) \in[0, T] \times \mathbb{R}^{d}, s \in[0, t]$ that $\mathbb{E}\left[\left|U_{n, M, Q}^{0}\left(t, z+W_{s}^{0}\right)\right|\right]<\infty$. The triangle inequality, Lemma 3.4, Lemma 3.6, Lemma 3.8, Lemma 3.5, and Lemma3.9 imply that for all $n \in \mathbb{N}$, $k \in\{0,1, \ldots, 2 Q-1\}$ it holds that

$$
\begin{aligned}
& \left\|U_{n, M, Q}^{0}-\mathbb{E}\left[U_{n, M, Q}^{0}\right]\right\|_{k, Q} \\
& \leq \|[0, T] \times \mathbb{R}^{d} \ni(t, z) \mapsto M^{-n} \sum_{i=1}^{M^{n}}\left(g\left(z+W_{T}^{(0,0,-i)}-W_{t}^{(0,0,-i)}\right)\right. \\
& \left.-\mathbb{E}\left[g\left(z+W_{T}^{(0,0,-i)}-W_{t}^{(0,0,-i)}\right)\right]\right) \in \mathbb{R} \|_{k, Q} \\
& +\sum_{l=0}^{n-1} \|[0, T] \times \mathbb{R}^{d} \ni(t, z) \mapsto M^{l-n} \sum_{i=1}^{M^{n-l}} \sum_{r \in[t, T]} q^{Q,[t, T]}(r)\left(F\left(U_{l, M, Q}^{(0, l, i, r)}\right)\right. \\
& \left.-\mathbb{1}_{\mathbb{N}}(l) F\left(U_{l-1, M, Q}^{(0,-l, i, r)}\right)\right)\left(r, z+W_{r}^{(0, l, i)}-W_{t}^{(0, l, i)}\right) \\
& -M^{l-n} \sum_{i=1}^{M^{n-l}} \sum_{r \in[t, T]} q^{Q,[t, T]}(r) \mathbb{E}\left[\left(F\left(U_{l, M, Q}^{(0, l, i, r)}\right)\right.\right. \\
& \left.\left.-\mathbb{1}_{\mathbb{N}}(l) F\left(U_{l-1, M, Q}^{(0,-l, i, r)}\right)\right)\left(r, z+W_{r}^{(0, l, i)}-W_{t}^{(0, l, i)}\right)\right] \in \mathbb{R} \|_{k, Q}
\end{aligned}
$$




$$
\begin{aligned}
& \leq \frac{1}{\sqrt{M^{n}}}\left\|[0, T] \times \mathbb{R}^{d} \ni(t, z) \mapsto g\left(z+W_{T}^{0}-W_{t}^{0}\right) \in \mathbb{R}\right\|_{k, Q} \\
& +\sum_{l=0}^{n-1} \frac{1}{\sqrt{M^{n-l}}} \|[0, T] \times \mathbb{R}^{d} \ni(t, z) \mapsto \sum_{r \in[t, T]} q^{Q,[t, T]}(r) \\
& \cdot\left(F\left(U_{l, M, Q}^{(0,1,1, r)}\right)-\mathbb{1}_{\mathbb{N}}(l) F\left(U_{l-1, M, Q}^{(0,-1,1, r)}\right)\right)\left(r, z+W_{r}^{0}-W_{t}^{0}\right) \in \mathbb{R} \|_{k, Q} \\
& \leq \frac{1}{\sqrt{M^{n}}} \sup _{z \in \mathbb{R}^{d}} \sup _{s \in[0, T]}\left\|g\left(z+W_{s}^{0}\right)\right\|_{L^{2}(\mathbb{P} ; \mathbb{R})}\|1\|_{k, Q} \\
& +\sum_{l=0}^{n-1} \frac{1}{\sqrt{M^{n-l}}}\left\|F\left(U_{l, M, Q}^{(0,1,1,0)}\right)-\mathbb{1}_{\mathbb{N}}(l) F\left(U_{l-1, M, Q}^{(0,-1,1,0)}\right)\right\|_{k+1, Q} \\
& \leq \frac{1}{\sqrt{M^{n}}} \sup _{z \in \mathbb{R}^{d}} \sup _{s \in[0, T]}\left\|g\left(z+W_{s}^{0}\right)\right\|_{L^{2}(\mathbb{P} ; \mathbb{R})} \frac{T^{k}}{k !}+\frac{1}{\sqrt{M^{n}}}\|F(0)\|_{k+1, Q} \\
& +L \sum_{l=1}^{n-1} \frac{1}{\sqrt{M^{n-l}}}\left\|U_{l, M, Q}^{(0,1,1,0)}-U_{l-1, M, Q}^{(0,-1,1,0)}\right\|_{k+1, Q} \\
& \leq \frac{1}{\sqrt{M^{n}}} \sup _{z \in \mathbb{R}^{d}} \sup _{s \in[0, T]}\left\|g\left(z+W_{s}^{0}\right)\right\|_{L^{2}(\mathbb{P} ; \mathbb{R})} \frac{T^{k}}{k !} \\
& +\frac{1}{\sqrt{M^{n}}} \sup _{z \in \mathbb{R}^{d}} \sup _{r, s \in[0, T]}\left\|(F(0))\left(r, z+W_{s}^{0}\right)\right\|_{L^{2}(\mathbb{P} ; \mathbb{R})} \frac{T^{k+1}}{(k+1) !} \\
& +L \sum_{l=0}^{n-1}\left(\frac{\mathbb{1}_{(0, n)}(l)}{\sqrt{M^{n-l}}}+\frac{\mathbb{1}_{(-\infty, n-1)}(l)}{\sqrt{M^{n-l-1}}}\right)\left\|U_{l, M, Q}^{0}-u^{\infty}\right\|_{k+1, Q} .
\end{aligned}
$$

Next we analyze the time discretization error. Item(ii) of Lemma 3.10 and Item(ii) of Lemma 3.11 ensure that for all $n \in \mathbb{N}, s \in[0, T], z \in \mathbb{R}^{d}$ it holds that

$$
\begin{aligned}
\mathbb{E} & {\left[U_{n, M, Q}^{0}(s, z)\right]-u^{\infty}(s, z) } \\
& =\mathbb{E}\left[\sum_{t \in[s, T]} q^{Q,[s, T]}(t)\left(F\left(U_{n-1, M, Q}^{0}\right)\right)\left(t, z+W_{t-s}^{0}\right)-\int_{s}^{T}\left(F\left(u^{\infty}\right)\right)\left(t, z+W_{t-s}^{0}\right) d t\right] .
\end{aligned}
$$

This, the triangle inequality, Lemma 3.7, the fact that for all $u \in \mathcal{M}\left(\mathcal{B}\left([0, T] \times \mathbb{R}^{d}\right), \mathcal{B}(\mathbb{R})\right)$, $k \in \mathbb{N}_{0}$ it holds that $\|u\|_{k, Q} \leq \sup _{s \in[0, T], t \in[0, s], z \in \mathbb{R}^{d}}\left\|u\left(s, z+W_{t}^{0}\right)\right\|_{L^{2}(\mathbb{P} ; \mathbb{R})}\|1\|_{k, Q}$, Lemma 3.6, Lemma 3.5, and Lemma 3.8 demonstrate for all $n \in \mathbb{N}, k \in\{0,1, \ldots, 2 Q-1\}$ that

$$
\begin{aligned}
& \left\|\mathbb{E}\left[U_{n, M, Q}^{0}\right]-u^{\infty}\right\|_{k, Q} \\
& \leq\left\|[0, T] \times \mathbb{R}^{d} \ni(s, z) \mapsto \mathbb{E}\left[\sum_{t \in[s, T]} q^{Q,[s, T]}(t)\left(F\left(U_{n-1, M, Q}^{0}\right)-F\left(u^{\infty}\right)\right)\left(t, z+W_{t-s}^{0}\right)\right] \in \mathbb{R}\right\|_{k, Q}
\end{aligned}
$$




$$
\begin{aligned}
& +\|[0, T] \times \mathbb{R}^{d} \ni(s, z) \mapsto \mathbb{E}\left[\sum_{t \in[s, T]} q^{Q,[s, T]}(t)\left(F\left(u^{\infty}\right)\right)\left(t, z+W_{t-s}^{0}\right)\right. \\
& \left.\quad-\int_{s}^{T}\left(F\left(u^{\infty}\right)\right)\left(t, z+W_{t-s}^{0}\right) d t\right] \in \mathbb{R} \|_{k, Q} \\
& \leq \|[0, T] \times \mathbb{R}^{d} \ni(s, z) \mapsto \sum_{t \in[s, T]} q^{Q,[s, T]}(t)\left(F\left(U_{n-1, M, Q}^{0}\right)\right. \\
& \left.\quad-F\left(u^{\infty}\right)\right)\left(t, z+W_{t}^{0}-W_{s}^{0}\right) \in \mathbb{R}\left\|_{k, Q}+\varepsilon\right\| 1 \|_{k, Q} \\
& \leq\left\|F\left(U_{n-1, M, Q}^{0}\right)-F\left(u^{\infty}\right)\right\|_{k+1, Q}+\varepsilon\|1\|_{k, Q} \\
& \leq L\left\|U_{n-1, M, Q}^{0}-u^{\infty}\right\|_{k+1, Q}+\varepsilon \frac{T^{k}}{k !} .
\end{aligned}
$$

In the next step we combine the established bounds for the Monte Carlo error and the time discretization error to obtain a bound for the global error. More formally, observe that (50) and (52) ensure that for all $n \in \mathbb{N}, k \in\{0,1, \ldots, 2 Q-1\}$ it holds that

$$
\begin{aligned}
& \left\|U_{n, M, Q}^{0}-u^{\infty}\right\|_{k, Q} \leq\left\|U_{n, M, Q}^{0}-\mathbb{E}\left[U_{n, M, Q}^{0}\right]\right\|_{k, Q}+\left\|\mathbb{E}\left[U_{n, M, Q}^{0}\right]-u^{\infty}\right\|_{k, Q} \\
& \leq \frac{1}{\sqrt{M^{n}}} \frac{T^{k}}{k !}\left[\sup _{z \in \mathbb{R}^{d}} \sup _{s \in[0, T]}\left\|g\left(z+W_{s}^{0}\right)\right\|_{L^{2}(\mathbb{P} ; \mathbb{R})}\right. \\
& \left.\quad+T \sup _{z \in \mathbb{R}^{d}} \sup _{r, u \in[0, T]}\left\|(F(0))\left(r, z+W_{u}^{0}\right)\right\|_{L^{2}(\mathbb{P} ; \mathbb{R})}\right] \\
& \quad+L \sum_{l=0}^{n-1}\left(\frac{\mathbb{1}_{(0, n)}(l)}{\sqrt{M^{n-l-1}}}+\frac{\mathbb{1}_{(-\infty, n-1)}(l)}{\sqrt{M^{n-l-1}}}\right)\left\|U_{l, M, Q}^{0}-u^{\infty}\right\|_{k+1, Q} \\
& \quad+L\left\|U_{n-1, M, Q}^{0}-u^{\infty}\right\|_{k+1, Q}+\varepsilon \frac{T^{k}}{k !} \\
& =\frac{1}{\sqrt{M^{n}}} \frac{T^{k}}{k !} \sup _{z \in \mathbb{R}^{d}} \sup _{s \in[0, T]}\left\|g\left(z+W_{s}^{0}\right)\right\|_{L^{2}(\mathbb{P} ; \mathbb{R})} \\
& \quad+T \sup _{z \in \mathbb{R}^{d} r, u \in[0, T]} \sup _{\left.\left\|(F(0))\left(r, z+W_{u}^{0}\right)\right\|_{L^{2}(\mathbb{P} ; \mathbb{R})}\right]}\|\|_{u^{\infty} \|_{k+1, Q}}^{\sqrt{M^{n-1}}}+2 L \sum_{l=1}^{n-1} \frac{1}{\sqrt{M^{n-l-1}}}\left\|U_{l, M, Q}^{0}-u^{\infty}\right\|_{k+1, Q}+\varepsilon \frac{T^{k}}{k !} .
\end{aligned}
$$

Hence, we obtain that for all $j \in \mathbb{N}_{0}, n \in \mathbb{N}, k \in\{0,1, \ldots, 2 Q-1\}$ it holds that

$$
\begin{aligned}
& \sqrt{M^{-j}}\left\|U_{n, M, Q}^{0}-u^{\infty}\right\|_{k, Q} \leq \frac{L\left\|u^{\infty}\right\|_{k+1, Q}}{\sqrt{M^{n+j-1}}}+\varepsilon \frac{T^{k}}{k ! \sqrt{M^{j}}} \\
& +2 L \sum_{l=1}^{n-1} \sqrt{M^{-j-n+l+1}}\left\|U_{l, M, Q}^{0}-u^{\infty}\right\|_{k+1, Q}
\end{aligned}
$$




$$
\begin{aligned}
& +\frac{T^{k}}{k ! \sqrt{M^{j+n}}}\left[\sup _{z \in \mathbb{R}^{d}} \sup _{s \in[0, T]}\left\|g\left(z+W_{s}^{0}\right)\right\|_{L^{2}(\mathbb{P} ; \mathbb{R})}\right. \\
& \left.+T \sup _{z \in \mathbb{R}^{d}} \sup _{r, u \in[0, T]}\left\|(F(0))\left(r, z+W_{u}^{0}\right)\right\|_{L^{2}(\mathbb{P} ; \mathbb{R})}\right] .
\end{aligned}
$$

This shows for all $n \in\{1,2, \ldots, N\}$ that

$$
\begin{aligned}
e_{n} \leq & L \sup _{k \in\{0,1, \ldots, N-1\}} \frac{\left\|u^{\infty}\right\|_{k+1, Q}}{\sqrt{M^{N-k-1}}}+\varepsilon e^{T}+2 L \sum_{l=1}^{n-1} e_{l} \\
+ & \left.\quad \sup _{i \in\{0,1, \ldots, N-1\}} \frac{T^{i}}{i ! \sqrt{M^{N-i}}}\right]\left[\sup _{z \in \mathbb{R}^{d}} \sup _{s \in[0, T]}\left\|g\left(z+W_{s}^{0}\right)\right\|_{L^{2}(\mathbb{P} ; \mathbb{R})}\right. \\
& \left.+T \sup _{z \in \mathbb{R}^{d}} \sup _{r, u \in[0, T]}\left\|(F(0))\left(r, z+W_{u}^{0}\right)\right\|_{L^{2}(\mathbb{P} ; \mathbb{R})}\right] .
\end{aligned}
$$

Combining this with the discrete Gronwall-type inequality in Agarwal [1, Corollary 4.1.2] proves that

$$
\begin{gathered}
\left\|U_{N, M, Q}^{0}-u^{\infty}\right\|_{0, Q}=e_{N} \leq(1+2 L)^{N-1}\left\{L \sup _{i \in\{1,2, \ldots, N\}} \frac{\left\|u^{\infty}\right\|_{i, Q}}{\sqrt{M^{N-i}}}+\varepsilon e^{T}\right. \\
+\left[\sup _{i \in\{0,1, \ldots, N-1\}} \frac{T^{i}}{i ! \sqrt{M^{N-i}}}\right]\left[\sup _{z \in \mathbb{R}^{d}} \sup _{s \in[0, T]}\left\|g\left(z+W_{s}^{0}\right)\right\|_{L^{2}(\mathbb{P} ; \mathbb{R})}\right. \\
\left.\left.+T \sup _{z \in \mathbb{R}^{d}} \sup _{r, u \in[0, T]}\left\|(F(0))\left(r, z+W_{u}^{0}\right)\right\|_{L^{2}(\mathbb{P} ; \mathbb{R})}\right]\right\} .
\end{gathered}
$$

This completes the proof of Theorem 3.12.

In the proof of the following result, Corollary 3.13, an upper bound for the quadrature error on the right-hand side of (48) is derived under the hypothesis that the solution of the PDE is sufficiently smooth and regular.

Corollary 3.13 Assume the setting in Sect.3.1, assume that $u^{\infty} \in C^{\infty}\left([0, T] \times \mathbb{R}^{d}, \mathbb{R}\right)$, assume for all $k \in \mathbb{N}_{0}, x \in \mathbb{R}^{d}, t \in[0, T]$ that

$$
\mathbb{E}\left[\sup _{s \in[t, T]}\left|\left(\left(\frac{\partial}{\partial r}+\frac{1}{2} \Delta_{y}\right)^{k} u^{\infty}\right)\left(s, x+W_{s-t}^{0}\right)\right|\right]<\infty,
$$

and let $M, Q \in \mathbb{N}, N \in\{1, \ldots, 2 Q-1\}$. Then it holds for all $\theta \in \Theta$ that

$$
\begin{aligned}
& \left\|U_{N, M, Q}^{\theta}-u^{\infty}\right\|_{0, Q} \leq(1+2 L)^{N-1}\left\{\sup _{i \in\{1,2, \ldots, N\}} \frac{\left\|u^{\infty}\right\|_{i, Q}}{\sqrt{M^{N-i}}}\right. \\
& +\left[\sup _{i \in\{0,1, \ldots, N-1\}} \frac{T^{i}}{i !{\sqrt{M^{N-i}}}^{\prime}}\right]\left[\sup _{z \in \mathbb{R}^{d}} \sup _{s \in[0, T]}\left\|g\left(z+W_{s}^{0}\right)\right\|_{L^{2}(\mathbb{P} ; \mathbb{R})}\right. \\
& \left.+T \sup _{z \in \mathbb{R}^{d}} \sup _{r, u \in[0, T]}\left\|(F(0))\left(r, z+W_{u}^{0}\right)\right\|_{L^{2}(\mathbb{P} ; \mathbb{R})}\right] \\
& +e^{T} \sup _{t \in[0, T]}\left[\sup _{u \in[0, t]} \sup _{z \in \mathbb{R}^{d}} \| \sup _{s \in[t, T]} \mid \mathbb{E}\left[\left(\left(\frac{\partial}{\partial r}+\frac{1}{2} \Delta_{y}\right)^{2 Q+1} u^{\infty}\right)\right.\right. \\
& \left.\left.\left.\cdot\left(s, x+W_{s-t}^{0}\right)\right]\left\|\left.\right|_{x=z+W_{u}^{0}}\right\|_{L^{2}(\mathbb{P} ; \mathbb{R})} \frac{[Q !]^{4}(T-t)^{2 Q+1}}{(2 Q+1)[(2 Q) !]^{3}}\right]\right\} .
\end{aligned}
$$


Proof Throughout this proof assume w.l.o.g. that $\sup _{z \in \mathbb{R}^{d}} \sup _{t, s \in[0, T]} \mathbb{E}\left[\left|g\left(z+W_{t}^{0}\right)\right|+\right.$ $\left.\left|(F(0))\left(t, z+W_{s}^{0}\right)\right|\right]<\infty$ (otherwise the right-hand side of (58) is infinite and the proof of (58) is clear). Observe that (57) and the dominated convergence theorem ensure that for every $k \in \mathbb{N}_{0}, x \in \mathbb{R}^{d}, t \in[0, T]$ it holds that the function

$$
[t, T] \ni s \mapsto \mathbb{E}\left[\left(\left(\frac{\partial}{\partial r}+\frac{1}{2} \Delta_{y}\right)^{k} u^{\infty}\right)\left(s, x+W_{s-t}^{0}\right)\right] \in \mathbb{R}
$$

is continuous. The assumption that $u^{\infty} \in C^{\infty}\left([0, T] \times \mathbb{R}^{d}, \mathbb{R}\right)$ and Itô's formula imply that for all $x \in \mathbb{R}^{d}, t \in[0, T], s \in[t, T], k \in \mathbb{N}$ it holds $\mathbb{P}$-a.s. that

$$
\begin{aligned}
& \left(\left(\frac{\partial}{\partial r}+\frac{1}{2} \Delta_{y}\right)^{k} u^{\infty}\right)\left(s, x+W_{s}^{0}-W_{t}^{0}\right)-\left(\left(\frac{\partial}{\partial r}+\frac{1}{2} \Delta_{y}\right)^{k} u^{\infty}\right)(t, x) \\
& =\int_{t}^{s}\left(\left(\frac{\partial}{\partial r}+\frac{1}{2} \Delta_{y}\right)^{k+1} u^{\infty}\right)\left(v, x+W_{v}^{0}-W_{t}^{0}\right) d v \\
& \quad+\int_{t}^{s}\left\langle\left(\nabla_{y}\left(\frac{\partial}{\partial r}+\frac{1}{2} \Delta_{y}\right)^{k} u^{\infty}\right)\left(v, x+W_{v}^{0}-W_{t}^{0}\right), d W_{v}^{0}\right\rangle .
\end{aligned}
$$

This and (57) show that for all $x \in \mathbb{R}^{d}, t \in[0, T], k \in \mathbb{N}$ it holds that $\mathbb{E}\left[\sup _{s \in[t, T]}\left|\int_{t}^{s}\left\langle\left(\nabla_{y}\left(\frac{\partial}{\partial r}+\frac{1}{2} \Delta_{y}\right)^{k} u^{\infty}\right)\left(v, x+W_{v}^{0}-W_{t}^{0}\right), d W_{v}^{0}\right\rangle\right|\right]<\infty$. This and the dominated convergence theorem imply that for all $x \in \mathbb{R}^{d}, t \in[0, T], s \in[t, T], k \in \mathbb{N}$ it holds that $\mathbb{E}\left[\int_{t}^{s}\left\langle\left(\nabla_{y}\left(\frac{\partial}{\partial r}+\frac{1}{2} \Delta_{y}\right)^{k} u^{\infty}\right)\left(v, x+W_{v}^{0}-W_{t}^{0}\right), d W_{v}^{0}\right\rangle\right]=0$. This, (60), and Fubini's theorem show that for all $x \in \mathbb{R}^{d}, t \in[0, T], s \in[t, T], k \in \mathbb{N}$ it holds that

$$
\begin{aligned}
\mathbb{E} & {\left[\left(\left(\frac{\partial}{\partial r}+\frac{1}{2} \Delta_{y}\right)^{k} u^{\infty}\right)\left(s, x+W_{s}^{0}-W_{t}^{0}\right)\right]-\left(\left(\frac{\partial}{\partial r}+\frac{1}{2} \Delta_{y}\right)^{k} u^{\infty}\right)(t, x) } \\
& =\int_{t}^{s} \mathbb{E}\left[\left(\left(\frac{\partial}{\partial r}+\frac{1}{2} \Delta_{y}\right)^{k+1} u^{\infty}\right)\left(v, x+W_{v}^{0}-W_{t}^{0}\right)\right] d v .
\end{aligned}
$$

Equation(61) (with $k=1$ ) together with (59) (with $k=2$ ) implies for every $x \in \mathbb{R}^{d}$, $t \in[0, T)$ that the function $[t, T] \ni s \mapsto \mathbb{E}\left[\left(\left(\frac{\partial}{\partial r}+\frac{1}{2} \Delta_{y}\right) u^{\infty}\right)\left(s, x+W_{s}^{0}-W_{t}^{0}\right)\right] \in \mathbb{R}$ is continuously differentiable. Induction, (59), and (61) prove that for every $x \in \mathbb{R}^{d}, t \in[0, T]$ it holds that the function $[t, T] \ni s \mapsto \mathbb{E}\left[\left(\left(\frac{\partial}{\partial r}+\frac{1}{2} \Delta_{y}\right) u^{\infty}\right)\left(s, x+W_{s}^{0}-W_{t}^{0}\right)\right] \in \mathbb{R}$ is infinitely often differentiable. This, induction, and (61) demonstrate that for all $k \in \mathbb{N}$, $x \in \mathbb{R}^{d}, t \in[0, T), s \in[t, T]$ it holds that

$$
\frac{\partial^{k}}{\partial s^{k}} \mathbb{E}\left[\left(\left(\frac{\partial}{\partial r}+\frac{1}{2} \Delta_{y}\right) u^{\infty}\right)\left(s, x+W_{s}^{0}-W_{t}^{0}\right)\right]=\mathbb{E}\left[\left(\left(\frac{\partial}{\partial r}+\frac{1}{2} \Delta_{y}\right)^{k+1} u^{\infty}\right)\left(s, x+W_{s}^{0}-W_{t}^{0}\right)\right] .
$$

Equation (15) and the error representation for the Gauß-Legendre quadrature rule (see, e.g., [15, Display (2.7.12)]) imply for all $x \in \mathbb{R}^{d}, t \in[0, T)$ that there exists a real number $\xi \in[t, T]$ such that

$$
\begin{aligned}
& \sum_{s \in[t, T]} q^{Q,[t, T]}(s) \mathbb{E}\left[\left(F\left(u^{\infty}\right)\right)\left(s, x+W_{s}^{0}-W_{t}^{0}\right)\right]-\int_{t}^{T} \mathbb{E}\left[\left(F\left(u^{\infty}\right)\right)\left(s, x+W_{s}^{0}-W_{t}^{0}\right)\right] d s \\
& =\int_{t}^{T} \mathbb{E}\left[\left(\left(\frac{\partial}{\partial r}+\frac{1}{2} \Delta_{y}\right) u^{\infty}\right)\left(s, x+W_{s}^{0}-W_{t}^{0}\right)\right] d s \\
& \quad-\sum_{s \in[t, T]} q^{Q,[t, T]}(s) \mathbb{E}\left[\left(\left(\frac{\partial}{\partial r}+\frac{1}{2} \Delta_{y}\right) u^{\infty}\right)\left(s, x+W_{s}^{0}-W_{t}^{0}\right)\right] \\
& =\left.\left(\frac{\partial^{2} Q}{\partial s^{2} Q} \mathbb{E}\left[\left(\left(\frac{\partial}{\partial r}+\frac{1}{2} \Delta_{y}\right) u^{\infty}\right)\left(s, x+W_{s}^{0}-W_{t}^{0}\right)\right]\right)\right|_{s=\xi} \frac{[Q !]^{4}(T-t)^{2 Q+1}}{(2 Q+1)[(2 Q) !]^{3}} .
\end{aligned}
$$


This and (62) prove that

$$
\begin{aligned}
& \sup _{\substack{t \in[0, T], \mathbb{R}_{z \in[0, t]} \\
z \in \mathbb{R}^{d}}} \| \mathbb{E}\left[\sum_{s \in[t, T]} q^{Q,[t, T]}(s)\left(F\left(u^{\infty}\right)\right)\left(s, z+W_{u+s-t}^{0}\right)\right. \\
& \left.\quad-\int_{t}^{T}\left(F\left(u^{\infty}\right)\right)\left(s, z+W_{u+s-t}^{0}\right) d s \mid W_{u}^{0}\right] \|_{L^{2}(\mathbb{P} ; \mathbb{R})} \\
& \leq \sup _{t \in[0, T)} \sup _{u \in[0, t]} \sup _{z \in \mathbb{R}^{d}}\left\{\| \sup _{s \in[t, T]} \mid \frac{\partial^{2 Q}}{\partial s^{2 Q}} \mathbb{E}\left[\left(\left(\frac{\partial}{\partial r}+\frac{1}{2} \Delta_{y}\right) u^{\infty}\right)\right.\right. \\
& \leq \sup _{t \in[0, T]} \sup _{u \in[0, t]} \sup _{z \in \mathbb{R}^{d}}\left\{\| \sup _{s \in[t, T]} \mid \mathbb{E}\left[\left(\left(\frac{\partial}{\partial r}+\frac{1}{2} \Delta_{y}\right)^{2 Q+1} u^{\infty}\right)\right.\right. \\
& \left.\left.\cdot\left(s, x+W_{s}^{0}-W_{t}^{0}\right)\right]\left.\right|_{x=z+W_{u}^{0}} \mid \|_{L^{2}(\mathbb{P} ; \mathbb{R})} \frac{[Q !]^{4}(T-t)^{2 Q+1}}{(2 Q+1)[(2 Q) !]^{3}}\right\} \\
&
\end{aligned}
$$

Theorem 3.12 together with (64) implies (58). The proof of Corollary 3.13 is thus completed.

The following result, Corollary 3.14, establishes an upper bound for the $L^{2}$-error between the solution of the PDE and our approximations (18) if the sup-norm of the $n$-th derivative of the solution of the PDE grows sufficiently slowly as $\mathbb{N} \ni n \rightarrow \infty$.

Corollary 3.14 Assume the setting in Sect.3.1, assume that $u^{\infty} \in C^{\infty}\left([0, T] \times \mathbb{R}^{d}, \mathbb{R}\right)$, let $\alpha \in\left[0, \frac{1}{4}\right]$, and let $C \in[0, \infty]$ be the extended real number given by

$$
\begin{aligned}
C=L & {\left[\sup _{(t, x) \in[0, T] \times \mathbb{R}^{d}}\left|u^{\infty}(t, x)\right|\right]+\left[\sup _{x \in \mathbb{R}^{d}}|g(x)|\right]+T\left[\sup _{(t, x) \in[0, T] \times \mathbb{R}^{d}}|(F(0))(t, x)|\right] } \\
& +T e^{T}\left[\sup _{k \in \mathbb{N}} \sup _{(t, x) \in[0, T] \times \mathbb{R}^{d}}(k !)^{\alpha-1}\left|\left(\left(\frac{\partial}{\partial r}+\frac{1}{2} \Delta_{y}\right)^{k} u^{\infty}\right)(t, x)\right|\right] .
\end{aligned}
$$

Then it holds for all $M, Q \in \mathbb{N}, N \in\{1, \ldots, 2 Q-1\}$ that

$\sup _{(t, x) \in[0, T] \times \mathbb{R}^{d}}\left\|U_{N, M, Q^{0}}^{0}(t, x)-u^{\infty}(t, x)\right\|_{L^{2}(\mathbb{P} ; \mathbb{R})} \leq C(1+2 L)^{N} \max \left\{\frac{T^{2 Q}}{Q^{2 \alpha Q}}, \frac{\exp (T \sqrt{M})}{M^{N / 2}}\right\}$.

Proof To prove (66) we assume w.l.o.g. that $C \in[0, \infty)$. Observe that the Stirling-type formula in Robbins [44, Displays (1)-(2)] proves for all $n \in \mathbb{N}$ that

$$
\sqrt{2 \pi n}\left[\frac{n}{e}\right]^{n} \leq n ! \leq \sqrt{2 \pi n}\left[\frac{n}{e}\right]^{n} e^{\frac{1}{12}}
$$

This together with the fact that $\sqrt{e} \leq 2$ and the fact that $\forall n \in \mathbb{N}: \pi e^{\frac{1}{3}} n \leq 8^{n}$ shows for all $n \in \mathbb{N}$ that 


$$
\begin{aligned}
\frac{n^{2 \alpha n}((2 n+1) !)^{1-\alpha}[n !]^{4}}{(2 n+1)[(2 n) !]^{3}} & \leq \frac{n^{2 \alpha n}[n !]^{4}}{[(2 n) !]^{2+\alpha}} \leq \frac{n^{2 \alpha n}\left[\sqrt{2 \pi} n^{n+\frac{1}{2}} e^{-n+\frac{1}{12}}\right]^{4}}{\left[\sqrt{2 \pi}(2 n)^{2 n+\frac{1}{2}} e^{-2 n}\right]^{2+\alpha}} \\
& =(\sqrt{2 \pi})^{2-\alpha} n^{1-\frac{\alpha}{2}} e^{\frac{1}{3}+2 n \alpha} 2^{-\left(2 n+\frac{1}{2}\right)(2+\alpha)} \\
& \leq 2 \pi n e^{\frac{1}{3}+\frac{n}{2}} 2^{-4 n-1}=\pi e^{\frac{1}{3}} n(\sqrt{e})^{n} 2^{-4 n} \leq \pi e^{\frac{1}{3}} n 2^{-3 n} \leq 1 .
\end{aligned}
$$

Next note that Lemma 3.2 and (19) imply that for all $Q \in \mathbb{N}, i \in\{0,1, \ldots, 2 Q-1\}$ it holds that

$$
\left\|u^{\infty}\right\|_{i, Q} \leq\left[\sup _{(t, x) \in[0, T] \times \mathbb{R}^{d}}\left|u^{\infty}(t, x)\right|\right]\left[\sum_{s \in[0, T]} \bar{q}^{i, Q}(s)\right]=\left[\sup _{(t, x) \in[0, T] \times \mathbb{R}^{d}}\left|u^{\infty}(t, x)\right|\right] \frac{T^{i}}{i !}
$$

The assumption that $C \in[0, \infty)$ allows us to apply Corollary 3.13 to obtain for all $M, Q \in \mathbb{N}$, $N \in\{1, \ldots, 2 Q-1\}$ that

$$
\begin{aligned}
& \sup _{t \in[0, T]} \sup _{z \in \mathbb{R}^{d}}\left\|U_{N, M, Q^{0}}^{0}(t, z)-u^{\infty}(t, z)\right\|_{L^{2}(\mathbb{P} ; \mathbb{R})} \\
& \leq \sup _{t \in[0, T]} \sup _{z \in \mathbb{R}^{d}} \sup _{u \in[0, t]}\left\|U_{N, M, Q}^{0}\left(t, z+W_{u}^{0}\right)-u^{\infty}\left(t, z+W_{u}^{0}\right)\right\|_{L^{2}(\mathbb{P} ; \mathbb{R})}=\left\|U_{N, M, Q}^{0}-u^{\infty}\right\|_{0, Q} \\
& \leq(1+2 L)^{N-1}\left\{L \sup _{(t, x) \in[0, T] \times \mathbb{R}^{d}}\left|u^{\infty}(t, x)\right| \sup _{i \in\{0,1, \ldots, N\}} \frac{T^{i}}{i ! \sqrt{M^{N-i}}}\right. \\
& +\sup _{i \in\{0,1, \ldots, N\}} \frac{T^{i}}{i ! \sqrt{M^{N-i}}} \sup _{z \in \mathbb{R}^{d}}\left[\sup _{s \in[0, T]}\left\|g\left(z+W_{s}^{0}\right)\right\|_{L^{2}(\mathbb{P} ; \mathbb{R})}\right. \\
& \left.+T \sup _{r, u \in[0, T]}\left\|(F(0))\left(r, z+W_{u}^{0}\right)\right\|_{L^{2}(\mathbb{P} ; \mathbb{R})}\right] \\
& +e^{T} \sup _{t \in[0, T]} \sup _{u \in[0, t]} \sup _{z \in \mathbb{R}^{d}} \| \sup _{s \in[t, T]} \mid \mathbb{E}\left[\left(\left(\frac{\partial}{\partial r}+\frac{1}{2} \Delta_{y}\right)^{2 Q+1} u^{\infty}\right)\right. \\
& \left.\left.\cdot\left(s, x+W_{s-t}^{0}\right)\right]||_{x=z+W_{u}^{0}} \|_{L^{2}(\mathbb{P} ; \mathbb{R})} \frac{[Q !]^{4}(T-t)^{2 Q+1}}{(2 Q+1)[(2 Q) !]^{3}}\right\} \\
& \leq(1+2 L)^{N}\left\{e^{T} T^{2 Q+1} \sup _{(t, x) \in[0, T] \times \mathbb{R}^{d}}\left|\left(\left(\frac{\partial}{\partial r}+\frac{1}{2} \Delta_{y}\right)^{2 Q+1} u^{\infty}\right)(t, x)\right| \frac{[Q !]^{4}}{(2 Q+1)[(2 Q) !]^{3}}\right. \\
& +\frac{1}{\sqrt{M^{N}}} \sup _{i \in\{0,1, \ldots, N\}}\left(\frac{(\sqrt{M} T)^{i}}{i !}\right)\left[L \sup _{(t, x) \in[0, T] \times \mathbb{R}^{d}}\left|u^{\infty}(t, x)\right|+\sup _{x \in \mathbb{R}^{d}}|g(x)|\right. \\
& \left.\left.+T \sup _{(t, x) \in[0, T] \times \mathbb{R}^{d}}|(F(0))(t, x)|\right]\right\} \text {. }
\end{aligned}
$$

This, (68), and the fact that $\sup _{i \in\{0,1, \ldots, N\}} \frac{(\sqrt{M} T)^{i}}{i !} \leq e^{T \sqrt{M}}$ imply for all $M, Q \in \mathbb{N}, N \in$ $\{1, \ldots, 2 Q-1\}$ that

$$
\begin{aligned}
& \sup _{(t, x) \in[0, T] \times \mathbb{R}^{d}}\left\|U_{N, M, Q}^{0}(t, x)-u^{\infty}(t, x)\right\|_{L^{2}(\mathbb{P} ; \mathbb{R})} \\
& \leq \frac{(1+2 L)^{N}}{Q^{2 \alpha Q}} e^{T} T^{2 Q+1}\left[\sup _{k \in \mathbb{N}} \sup _{(t, x) \in[0, T] \times \mathbb{R}^{d}}(k !)^{\alpha-1}\left|\left(\left(\frac{\partial}{\partial r}+\frac{1}{2} \Delta_{y}\right)^{k} u^{\infty}\right)(t, x)\right|\right]
\end{aligned}
$$




$$
\begin{aligned}
& \cdot\left[\sup _{n \in \mathbb{N}} \frac{n^{2 \alpha n}((2 n+1) !)^{1-\alpha}[n !]^{4}}{(2 n+1)[(2 n) !]^{3}}\right] \\
& +\left(\frac{1+2 L}{\sqrt{M}}\right)^{N} e^{T \sqrt{M}}\left[L \sup _{(t, x) \in[0, T] \times \mathbb{R}^{d}}\left|u^{\infty}(t, x)\right|+\sup _{x \in \mathbb{R}^{d}}|g(x)|\right. \\
& \left.+T \sup _{(t, x) \in[0, T] \times \mathbb{R}^{d}}|(F(0))(t, x)|\right] \\
& \leq \frac{(1+2 L)^{N}}{Q^{2 \alpha Q}} e^{T} T^{2 Q+1}\left[\sup _{k \in \mathbb{N}} \sup _{(t, x) \in[0, T] \times \mathbb{R}^{d}}(k !)^{\alpha-1}\left|\left(\left(\frac{\partial}{\partial r}+\frac{1}{2} \Delta_{y}\right)^{k} u^{\infty}\right)(t, x)\right|\right] \\
& +\left(\frac{1+2 L}{\sqrt{M}}\right)^{N} e^{T \sqrt{M}}\left[L \sup _{(t, x) \in[0, T] \times \mathbb{R}^{d}}\left|u^{\infty}(t, x)\right|+\sup _{x \in \mathbb{R}^{d}}|g(x)|\right. \\
& \left.+T \sup _{(t, x) \in[0, T] \times \mathbb{R}^{d}}|(F(0))(t, x)|\right] .
\end{aligned}
$$

This establishes (66). The proof of Corollary 3.14 is thus completed.

The next result, Corollary 3.15, provides an upper bound for the $L^{2}$-error between the solution of the PDE and our approximations (18) if the parameters $N, M, Q \in \mathbb{N}$ satisfy $N=M=Q$. Corollary 3.15 is a direct consequence of Corollary 3.14.

Corollary 3.15 Assume the setting in Sect.3.1, assume that $u^{\infty} \in C^{\infty}\left([0, T] \times \mathbb{R}^{d}, \mathbb{R}\right)$, let $\alpha \in\left[0, \frac{1}{4}\right]$, and let $C \in[0, \infty]$ be the extended real number given by

$$
\begin{aligned}
C=L & {\left[\sup _{(t, x) \in[0, T] \times \mathbb{R}^{d}}\left|u^{\infty}(t, x)\right|\right]+\left[\sup _{x \in \mathbb{R}^{d}}|g(x)|\right]+T\left[\sup _{(t, x) \in[0, T] \times \mathbb{R}^{d}}|(F(0))(t, x)|\right] } \\
& +T e^{T}\left[\sup _{k \in \mathbb{N}} \sup _{(t, x) \in[0, T] \times \mathbb{R}^{d}}(k !)^{\alpha-1}\left|\left(\left(\frac{\partial}{\partial r}+\frac{1}{2} \Delta_{y}\right)^{k} u^{\infty}\right)(t, x)\right|\right]
\end{aligned}
$$

Then it holds for all $N \in \mathbb{N}$ that

$$
\sup _{(t, x) \in[0, T] \times \mathbb{R}^{d}}\left\|U_{N, N, N}^{0}(t, x)-u^{\infty}(t, x)\right\|_{L^{2}(\mathbb{P} ; \mathbb{R})} \leq C\left[\frac{(1+2 L) e^{T}}{N^{2 \alpha}}\right]^{N} .
$$

\subsection{Analysis of the computational complexity and overall rate of convergence}

In Lemma 3.16 $\mathrm{RN}_{n, M, Q}$ is the number of realizations of a scalar standard normal random variable used to compute one realization of the random variable $U_{n, M, Q}^{\theta}(t, x): \Omega \rightarrow \mathbb{R}$. In Lemma 3.17 $\mathrm{FE}_{n, M, Q}$ is the number of function evaluations of $f$ and $g$ used to compute one realization of $U_{n, M, Q}^{\theta}(t, x): \Omega \rightarrow \mathbb{R}$.

Lemma 3.16 Assume the setting in Sect.3.1 and let $\left(\mathrm{RN}_{n, M, Q}\right)_{n, M, Q \in \mathbb{Z}} \subseteq \mathbb{N}_{0}$ be natural numbers which satisfy for all $n, M, Q \in \mathbb{N}$ that $\mathrm{RN}_{0, M, Q}=0$ and 


$$
\mathrm{RN}_{n, M, Q} \leq d M^{n}+\sum_{l=0}^{n-1}\left[Q M^{n-l}\left(d+\mathrm{RN}_{l, M, Q}+\mathbb{1}_{\mathbb{N}}(l) \cdot \mathrm{RN}_{l-1, M, Q}\right)\right] .
$$

Then for all $N \in \mathbb{N}$, we have

$$
\mathrm{RN}_{N, N, N} \leq 8 d N^{2 N}
$$

Proof Inequality (74) implies for all $n, Q \in \mathbb{N}, M \in \mathbb{N} \cap[2, \infty)$ that

$$
\begin{aligned}
\left(M^{-n} \cdot \mathrm{RN}_{n, M, Q}\right) & \leq d+\sum_{l=0}^{n-1}\left[Q M^{-l}\left(d+\mathrm{RN}_{l, M, Q}+\mathbb{1}_{\mathbb{N}}(l) \cdot \mathrm{RN}_{l-1, M, Q}\right)\right] \\
& \leq d\left(1+\frac{M Q}{M-1}\right)+\left(1+\frac{1}{M}\right) Q\left[\sum_{l=0}^{n-1}\left(M^{-l} \cdot \mathrm{RN}_{l, M, Q}\right)\right] .
\end{aligned}
$$

The fact that $\forall M, Q \in \mathbb{N}: \mathrm{RN}_{0, M, Q}=0$ and the discrete Gronwall-type inequality in Agarwal [1, Corollary 4.1.2] hence prove that for all $n, Q \in \mathbb{N}, M \in \mathbb{N} \cap[2, \infty)$ it holds that

$$
\left(M^{-n} \cdot \mathrm{RN}_{n, M, Q}\right) \leq d\left(1+\frac{M Q}{M-1}\right)\left(1+\left(1+\frac{1}{M}\right) Q\right)^{n-1} \leq \frac{d(M+(M+1) Q)^{n}}{M^{n-1}(M-1)} .
$$

Hence, we obtain that for all $N \in \mathbb{N} \cap[2, \infty)$ it holds that

$$
\mathrm{RN}_{N, N, N} \leq \frac{N d}{N-1}(N+(N+1) N)^{N}=\frac{N}{N-1}\left(1+\frac{2}{N}\right)^{N} d N^{2 N} \leq 8 d N^{2 N} .
$$

This and the fact that $\mathrm{RN}_{1,1,1} \leq 2 d$ complete the proof of Lemma 3.16.

Lemma 3.17 Assume the setting in Sect.3.1 and let $\left(\mathrm{FE}_{n, M, Q}\right)_{n, M, Q \in \mathbb{Z}} \subseteq \mathbb{N}_{0}$ be natural numbers which satisfy for all $n, M, Q \in \mathbb{N}$ that $\mathrm{FE}_{0, M, Q}=0$ and

$$
\mathrm{FE}_{n, M, Q} \leq M^{n}+\sum_{l=0}^{n-1}\left[Q M^{n-l}\left(1+\mathrm{FE}_{l, M, Q}+\mathbb{1}_{\mathbb{N}}(l)+\mathbb{1}_{\mathbb{N}}(l) \cdot \mathrm{FE}_{l-1, M, Q}\right)\right] .
$$

Then for all $N \in \mathbb{N}$, we have

$$
\mathrm{FE}_{N, N, N} \leq 8 N^{2 N}
$$

The proof of Lemma 3.17 is analogous to the proof of Lemma 3.16 and therefore omitted. In the proof of Corollary 3.18 below we combine Lemma 3.16 and Lemma 3.17 with Corollary 3.15 to obtain a bound for the computational complexity of the scheme (18) in terms of the space dimension and the prescribed approximation accuracy.

The following corollary, Corollary 3.18, proves under suitable assumptions for every $\alpha \in$ $\left(0, \frac{1}{4}\right]$ and every $\delta \in(0, \infty)$ that the computational effort of the approximation method (number of function evaluations of the coefficient functions of the considered PDE and number of used independent scalar standard normal random variables, cf. Section 3.7) is at most $O\left(d \varepsilon^{-\left(\frac{1}{\alpha}+\delta\right)}\right)$ where $\varepsilon \in(0, \infty)$ is the prescribed approximation accuracy and $d \in \mathbb{N}$ is the dimension of the considered PDE.

Corollary 3.18 Assume the setting in Sect.3.1, assume that $u^{\infty} \in C^{\infty}\left([0, T] \times \mathbb{R}^{d}, \mathbb{R}\right)$, let $\alpha \in\left(0, \frac{1}{4}\right], \delta \in(0, \infty)$, let $C \in[0, \infty)$, assume that 


$$
\begin{aligned}
C= & 16 \exp \left(2 \alpha \delta\left[e^{T}(1+2 L)\right]^{\frac{1+\alpha \delta}{2 \alpha^{2} \delta}}\right)\left\{L\left[\sup _{(t, x) \in[0, T] \times \mathbb{R}^{d}}\left|u^{\infty}(t, x)\right|\right]\right. \\
& +\left[\sup _{x \in \mathbb{R}^{d}}|g(x)|\right]+T\left[\sup _{(t, x) \in[0, T] \times \mathbb{R}^{d}}|(F(0))(t, x)|\right] \\
& \left.+T e^{T}\left[\sup _{k \in \mathbb{N}} \sup _{(t, x) \in[0, T] \times \mathbb{R}^{d}}(k !)^{\alpha-1}\left|\left(\left(\frac{\partial}{\partial r}+\frac{1}{2} \Delta_{y}\right)^{k} u^{\infty}\right)(t, x)\right|\right]\right\}^{\frac{1}{\alpha}+\delta},
\end{aligned}
$$

let $\left(\mathrm{RN}_{n, M, Q}\right)_{n, M, Q \in \mathbb{Z}} \subseteq \mathbb{N}_{0}$ be natural numbers which satisfy for all $n, M, Q \in \mathbb{N}$ that $\mathrm{RN}_{0, M, Q}=0$ and

$$
\mathrm{RN}_{n, M, Q} \leq d M^{n}+\sum_{l=0}^{n-1}\left[Q M^{n-l}\left(d+\mathrm{RN}_{l, M, Q}+\mathbb{1}_{\mathrm{N}}(l) \cdot \mathrm{RN}_{l-1, M, Q}\right)\right]
$$

(for every $N \in \mathbb{N}$ we think of $\mathrm{RN}_{N, N, N}$ as the number of realizations of a scalar standard normal random variable required to compute one realization of the random variable $\left.U_{N, N, N}^{0}(0,0): \Omega \rightarrow \mathbb{R}\right)$, and let $\left(\mathrm{FE}_{n, M, Q}\right)_{n, M, Q \in \mathbb{Z}} \subseteq \mathbb{N}_{0}$ be natural numbers which satisfy for all $n, M, Q \in \mathbb{N}$ that $\mathrm{FE}_{0, M, Q}=$ and

$$
\mathrm{FE}_{n, M, Q} \leq M^{n}+\sum_{l=0}^{n-1}\left[Q M^{n-l}\left(1+\mathrm{FE}_{l, M, Q}+\mathbb{1}_{\mathbb{N}}(l)+\mathbb{1}_{\mathbb{N}}(l) \cdot \mathrm{FE}_{l-1, M, Q}\right)\right]
$$

(for every $N \in \mathbb{N}$ we think of $\mathrm{FE}_{N, N, N}$ as the number of function evaluations of $f$ and $g$ required to compute one realization of the random variable $\left.U_{N, N, N}^{0}(0,0): \Omega \rightarrow \mathbb{R}\right)$. Then

(i) it holds that

$$
\limsup _{N \rightarrow \infty}\left[\sup _{(t, x) \in[0, T] \times \mathbb{R}^{d}}\left\|U_{N, N, N}^{0}(t, x)-u^{\infty}(t, x)\right\|_{L^{2}(\mathbb{P} ; \mathbb{R})}\right]=0
$$

and

(ii) it holds for all $N \in \mathbb{N}$ that

$$
\mathrm{RN}_{N, N, N}+\mathrm{FE}_{N, N, N} \leq C d\left[\sup _{(t, x) \in[0, T] \times \mathbb{R}^{d}}\left\|U_{N, N, N}^{0}(t, x)-u^{\infty}(t, x)\right\|_{L^{2}(\mathbb{P} ; \mathbb{R})}\right]^{-\left(\frac{1}{\alpha}+\delta\right)} .
$$

Proof Throughout this proof let $\tilde{C} \in[0, \infty)$ be the real number given by $\tilde{C}=$ $\frac{1}{16} \exp \left(-2 \alpha \delta\left[e^{T}(1+2 L)\right]^{\frac{1+\alpha \delta}{2 \alpha^{2} \delta}}\right) C$. First note that Corollary 3.15 proves item(i). Next observe that Corollary 3.15, Lemma 3.16, and Lemma 3.17 prove that for all $N \in \mathbb{N}$ it holds that

$$
\begin{aligned}
& \left(\mathrm{RN}_{N, N, N}+\mathrm{FE}_{N, N, N}\right)\left[\sup _{(t, x) \in[0, T] \times \mathbb{R}^{d}}\left\|U_{N, N, N}^{0}(t, x)-u^{\infty}(t, x)\right\|_{L^{2}(\mathbb{P} ; \mathbb{R})}\right]^{\frac{1}{\alpha}+\delta} \\
& \leq\left(8 d N^{2 N}+8 N^{2 N}\right) \tilde{C}\left[\frac{(1+2 L) e^{T}}{N^{2 \alpha}}\right]^{N\left(\frac{1}{\alpha}+\delta\right)}=8(d+1) \tilde{C}\left[(1+2 L) e^{T}\right]^{N\left(\frac{1}{\alpha}+\delta\right)} N^{-2 \alpha \delta N} .
\end{aligned}
$$


This and the fact that $\forall N \in \mathbb{N}: N ! \leq N^{N}$ show that for all $N \in \mathbb{N}$ it holds that

$$
\begin{aligned}
& \left(\mathrm{RN}_{N, N, N}+\mathrm{FE}_{N, N, N}\right)\left[\sup _{(t, x) \in[0, T] \times \mathbb{R}^{d}}\left\|U_{N, N, N}^{0}(t, x)-u^{\infty}(t, x)\right\|_{L^{2}(\mathbb{P} ; \mathbb{R})}\right]^{\frac{1}{\alpha}+\delta} \\
& \leq 16 d \tilde{C} \frac{\left[(1+2 L) e^{T}\right]^{N(}\left(\frac{1}{\alpha}+\delta\right)}{(N !)^{2 \alpha \delta}}=16 d \tilde{C}\left[\frac{\left[(1+2 L) e^{T}\right]^{N}\left(\frac{1+\alpha \delta}{2 \alpha^{2} \delta}\right)}{N !}\right]^{2 \alpha \delta} \\
& \leq 16 d \tilde{C}\left[\sum_{n=0}^{\infty} \frac{\left[(1+2 L) e^{T}\right]^{n\left(\frac{1+\alpha \delta}{2 \alpha^{2} \delta}\right)}}{n !}\right]^{2 \alpha \delta} \\
& =16 d \tilde{C}\left[\exp \left(\left[(1+2 L) e^{T}\right]^{\frac{1+\alpha \delta}{2 \alpha^{2} \delta}}\right)\right]^{2 \alpha \delta}=16 d \tilde{C}\left[\exp \left(2 \alpha \delta\left[(1+2 L) e^{T}\right]^{\frac{1+\alpha \delta}{2 \alpha^{2} \delta}}\right)\right]=C d
\end{aligned}
$$

This completes the proof of Corollary 3.18.

The next result, Corollary 3.19, specializes Corollary 3.18 to the case $\alpha=\frac{1}{4}$.

Corollary 3.19 Assume the setting in Sect.3.1, assume that $u^{\infty} \in C^{\infty}\left([0, T] \times \mathbb{R}^{d}, \mathbb{R}\right)$, let $\delta \in(0, \infty)$, let $C \in[0, \infty)$, assume that

$$
\begin{aligned}
C= & 16 \exp \left(\delta\left[e^{T}(1+2 L)\right]^{2+\left(\frac{8}{\delta}\right)}\right)\left\{L\left[\sup _{(t, x) \in[0, T] \times \mathbb{R}^{d}}\left|u^{\infty}(t, x)\right|\right]+\left[\sup _{x \in \mathbb{R}^{d}}|g(x)|\right]\right. \\
& +T\left[\sup _{(t, x) \in[0, T] \times \mathbb{R}^{d}}|(F(0))(t, x)|\right] \\
& \left.+T e^{T}\left[\sup _{k \in \mathbb{N}} \sup _{(t, x) \in[0, T] \times \mathbb{R}^{d}} \frac{\left|\left(\left(\frac{\partial}{\partial r}+\frac{1}{2} \Delta_{y}\right)^{k} u^{\infty}\right)(t, x)\right|}{(k !)^{\frac{3}{4}}}\right]\right\}^{4+\delta},
\end{aligned}
$$

let $\left(\mathrm{RN}_{n, M}, Q\right)_{n, M, Q \in \mathbb{Z}} \subseteq \mathbb{N}_{0}$ be natural numbers which satisfy for all $n, M, Q \in \mathbb{N}$ that $\mathrm{RN}_{0, M, Q}=0$ and

$$
\mathrm{RN}_{n, M, Q} \leq d M^{n}+\sum_{l=0}^{n-1}\left[Q M^{n-l}\left(d+\mathrm{RN}_{l, M, Q}+\mathbb{1}_{\mathbb{N}}(l) \cdot \mathrm{RN}_{l-1, M, Q}\right)\right]
$$

(for every $N \in \mathbb{N}$ we think of $\mathrm{RN}_{N, N, N}$ as the number of realizations of a scalar standard normal random variable required to compute one realization of the random variable $\left.U_{N, N, N}^{0}(0,0): \Omega \rightarrow \mathbb{R}\right)$, and let $\left(\mathrm{FE}_{n, M, Q}\right)_{n, M, Q \in \mathbb{Z}} \subseteq \mathbb{N}_{0}$ be natural numbers which satisfy for all $n, M, Q \in \mathbb{N}$ that $\mathrm{FE}_{0, M, Q}=$ and

$$
\mathrm{FE}_{n, M, Q} \leq M^{n}+\sum_{l=0}^{n-1}\left[Q M^{n-l}\left(1+\mathrm{FE}_{l, M, Q}+\mathbb{1}_{\mathbb{N}}(l)+\mathbb{1}_{\mathbb{N}}(l) \cdot \mathrm{FE}_{l-1, M, Q}\right)\right]
$$

(for every $N \in \mathbb{N}$ we think of $\mathrm{FE}_{N, N, N}$ as the number of function evaluations of $f$ and $g$ required to compute one realization of the random variable $\left.U_{N, N, N}^{0}(0,0): \Omega \rightarrow \mathbb{R}\right)$. Then

(i) it holds that

$$
\limsup _{N \rightarrow \infty}\left[\sup _{(t, x) \in[0, T] \times \mathbb{R}^{d}}\left\|U_{N, N, N}^{0}(t, x)-u^{\infty}(t, x)\right\|_{L^{2}(\mathbb{P} ; \mathbb{R})}\right]=0
$$


and

(ii) it holds for all $N \in \mathbb{N}$ that

$$
\mathrm{RN}_{N, N, N}+\mathrm{FE}_{N, N, N} \leq C d\left[\sup _{(t, x) \in[0, T] \times \mathbb{R}^{d}}\left\|U_{N, N, N}^{0}(t, x)-u^{\infty}(t, x)\right\|_{L^{2}(\mathbb{P} ; \mathbb{R})}\right]^{-(4+\delta)} .
$$

\subsection{An example PDE}

In this subsection we prove for a non-linear example that the assumptions of Corollary 3.19 are satisfied. For this, we construct an example with an explicit solution so that we can easily check condition (86). Throughout this subsection let $d \in \mathbb{N}$, let $g \in C^{\infty}\left(\mathbb{R}^{d}, \mathbb{R}\right)$, $u^{\infty}=\left(u^{\infty}(t, x)\right)_{(t, x) \in[0,1] \times \mathbb{R}^{d}} \in C^{\infty}\left([0,1] \times \mathbb{R}^{d}, \mathbb{R}\right), f:[0,1] \times \mathbb{R}^{d} \times \mathbb{R} \rightarrow \mathbb{R}$ be the functions which satisfy for all $(t, x, y) \in[0,1] \times \mathbb{R}^{d} \times \mathbb{R}$ that $f(t, x, y)=\min \left\{y^{2}, 1\right\}+$ $\cos \left(\frac{\sqrt{2}}{\sqrt{d}} \sum_{i=1}^{d} x_{i}\right)\left(1-\cos \left(\frac{\sqrt{2}}{\sqrt{d}} \sum_{i=1}^{d} x_{i}\right)\right)$ and $u^{\infty}(t, x)=g(x)=\cos \left(\frac{\sqrt{2}}{\sqrt{d}} \sum_{i=1}^{d} x_{i}\right)$. Then $f$ satisfies the Lipschitz-type condition(14) and it holds for all $(t, x) \in[0,1] \times \mathbb{R}^{d}$ that

$$
\begin{aligned}
\left(\frac{\partial}{\partial t}+\frac{1}{2} \Delta_{x}\right) u^{\infty}(t, x) & =\frac{1}{2} \Delta_{x} \cos \left(\frac{\sqrt{2}}{\sqrt{d}} \sum_{i=1}^{d} x_{i}\right)=-\cos \left(\frac{\sqrt{2}}{\sqrt{d}} \sum_{i=1}^{d} x_{i}\right) \\
& =-\left(u^{\infty}(t, x)\right)^{2}-\cos \left(\frac{\sqrt{2}}{\sqrt{d}} \sum_{i=1}^{d} x_{i}\right)\left(1-\cos \left(\frac{\sqrt{2}}{\sqrt{d}} \sum_{i=1}^{d} x_{i}\right)\right) \\
& =-\left(f\left(u^{\infty}\right)\right)(t, x)
\end{aligned}
$$

so that $u^{\infty}$ is a solution of the PDE(15). Moreover it holds for all $(t, x) \in[0,1] \times \mathbb{R}^{d}$, $k \in \mathbb{N}_{0}$ that

$$
\left(\left(\frac{\partial}{\partial t}+\frac{1}{2} \Delta_{x}\right)^{k} u^{\infty}\right)(t, x)=(-1)^{k} \cos \left(\frac{\sqrt{2}}{\sqrt{d}} \sum_{i=1}^{d} x_{i}\right)
$$

so that condition (86) is satisfied. Consequently, on a suitable probability space, Corollary 3.19 implies for every $\varepsilon \in(0,1 / 4)$ that there exist approximations of $u^{\infty}(0,0)$ which converge with $L^{2}$-convergence rate at least $1 / 4-\varepsilon$.

Acknowledgements This work has been funded by the Deutsche Forschungsgemeinschaft (DFG, German Research Foundation) under Germany's Excellence Strategy EXC 2044 - 390685587, Mathematics Münster: Dynamics - Geometry - Structure, by the Deutsche Forschungsgemeinschaft via research grant HU 1889/6-1, and through the research grants ONR N00014-13-1-0338 and DOE DE-SC0009248.

Open Access This article is licensed under a Creative Commons Attribution 4.0 International License, which permits use, sharing, adaptation, distribution and reproduction in any medium or format, as long as you give appropriate credit to the original author(s) and the source, provide a link to the Creative Commons licence, and indicate if changes were made. The images or other third party material in this article are included in the article's Creative Commons licence, unless indicated otherwise in a credit line to the material. If material is not included in the article's Creative Commons licence and your intended use is not permitted by statutory regulation or exceeds the permitted use, you will need to obtain permission directly from the copyright holder. To view a copy of this licence, visit http://creativecommons.org/licenses/by/4.0/. 


\section{References}

1. Agarwal, R.: Difference equations and inequalities: theory, methods, and applications. CRC Press, Chapman \& Hall (2000). (CRC Pure and Applied Mathematics)

2. Albeverio, S., Röckner, M.: Stochastic differential equations in infinite dimensions: solutions via Dirichlet forms. Probab. Theory Relat. Fields 89(3), 347-386 (1991)

3. Bally, V., Pagès, G.: A quantization algorithm for solving multi-dimensional discrete-time optimal stopping problems. Bernoulli 9(6), 1003-1049 (2003)

4. Beck, C., Hornung, F., Hutzenthaler, M., Jentzen, A., Kruse, T.: Overcoming the curse of dimensionality in the numerical approximation of Allen-Cahn partial differential equations via truncated full-history recursive multilevel Picard approximations. J. Numer. Math. 28(4), 197-222 (2020)

5. Bellman, R.: Dynamic programming. Princeton landmarks in mathematics, Princeton University Press, Princeton (2010). (Reprint of the 1957 edition, With a new introduction by Stuart Dreyfus)

6. Bender, C., Denk, R.: A forward scheme for backward SDEs. Stochastic Process. Appl. 117(12), 17931812 (2007)

7. Bergman, Y.Z.: Option pricing with differential interest rates. Rev. Financ. Stud. 8(2), 475-500 (1995)

8. Bouchard, B., Touzi, N.: Discrete-time approximation and Monte-Carlo simulation of backward stochastic differential equations. Stochastic Process. Appl. 111(2), 175-206 (2004)

9. Briand, P., Labart, C.: Simulation of BSDEs by Wiener chaos expansion. Ann. Appl. Probab. 24(3), 1129-1171 (2014)

10. Burgard, C., Kjaer, M.: Partial differential equation representations of derivatives with bilateral counterparty risk and funding costs. J. Credit Risk 7(3), 1-19 (2011)

11. Chassagneux, J.-F.: Linear multistep schemes for BSDEs. SIAM J. Numer. Anal. 52(6), 2815-2836(2014)

12. Creutzig, J., Dereich, S., Müller-Gronbach, T., Ritter, K.: Infinite-dimensional quadrature and approximation of distributions. Found. Comput. Math. 9(4), 391-429 (2009)

13. Crisan, D., Manolarakis, K.: Solving backward stochastic differential equations using the cubature method: application to nonlinear pricing. SIAM J. Financ. Math. 3(1), 534-571 (2012)

14. Da Prato, G., Zabczyk, J.: Differentiability of the Feynman-Kac semigroup and a control application. Atti Accad. Naz. Lincei Cl. Sci. Fis. Mat. Natur. Rend. Lincei (9) Mat. Appl. 8(3), 183-188 (1997)

15. Davis, P. J., Rabinowitz, P.: Methods of numerical integration. Courier Corpor. (2007)

16. Duffie, D., Schroder, M., Skiadas, C.: Recursive valuation of defaultable securities and the timing of resolution of uncertainty. Ann. Appl. Probab. 6(4), 1075-1090 (1996)

17. E, W., Hutzenthaler, M., Jentzen, A., Kruse, T.: On multilevel Picard numerical approximations for highdimensional nonlinear parabolic partial differential equations and high-dimensional nonlinear backward stochastic differential equations. J. Sci. Comput. 79(3), 1534-1571 (2019)

18. Elworthy, K., Li, X.-M.: Formulae for the derivatives of heat semigroups. J. Funct. Anal. 125(1), 252-286 (1994)

19. Giles, M.B.: Improved multilevel Monte Carlo convergence using the Milstein scheme. In Monte Carlo and quasi-Monte Carlo methods. Springer, Berlin 2008, 343-358 (2006)

20. Giles, M.B.: Multilevel Monte Carlo path simulation. Oper. Res. 56(3), 607-617 (2008)

21. Gobet, E., Labart, C.: Solving BSDE with adaptive control variate. SIAM J. Numer. Anal. 48(1), 257-277 (2010)

22. Gobet, E., Lemor, J.-P., Warin, X.: A regression-based Monte Carlo method to solve backward stochastic differential equations. Ann. Appl. Probab. 15(3), 2172-2202 (2005)

23. Graham, C., Talay, D.: Stochastic simulation and Monte Carlo methods, vol. 68 of Stochastic Modelling and Applied Probability. Springer, Heidelberg, (2013). Mathematical foundations of stochastic simulation

24. Gyöngy, I., Krylov, N.: Existence of strong solutions for Itô's stochastic equations via approximations. Probab. Theory Relat. Fields 105(2), 143-158 (1996)

25. Heinrich, S.: Monte Carlo complexity of global solution of integral equations. J. Complex. 14(2), 151-175 (1998)

26. Heinrich, S.: Multilevel Monte Carlo Methods. In Large-Scale Scientific Computing, vol. 2179 of Lecture Notes in Computer Science. Springer, pp. 58-67 (2001)

27. Henry-Labordère, P.: Counterparty risk valuation: a marked branching diffusion approach. arXiv:1203.2369, 17 pages (2012)

28. Henry-Labordère, P., Oudjane, N., Tan, X., Touzi, N., Warin, X.: Branching diffusion representation of semilinear PDEs and Monte Carlo approximation. Ann. Inst. H. Poincaré Probab. Statist. 55(1), 184-210 (2019)

29. Henry-Labordère, P., Tan, X., Touzi, N.: A numerical algorithm for a class of BSDEs via the branching process. Stochastic Process. Appl. 124(2), 1112-1140 (2014) 
30. Hutzenthaler, M., Jentzen, A.: On a perturbation theory and on strong convergence rates for stochastic ordinary and partial differential equations with non-globally monotone coefficients. Ann. Probab. 48(1), 53-93 (2020)

31. Hutzenthaler, M., Jentzen, A., Kloeden, P.E.: Strong convergence of an explicit numerical method for SDEs with nonglobally Lipschitz continuous coefficients. Ann. Appl. Probab. 22(4), 1611-1641 (2012)

32. Hutzenthaler, M., Jentzen, A., Kruse, T., Nguyen, T.A.: A proof that rectified deep neural networks overcome the curse of dimensionality in the numerical approximation of semilinear heat equations. SN Partial Differ. Equ. Appl. 1, 1-34 (2020)

33. Hutzenthaler, M., Jentzen, A., Kruse, T., Nguyen, T.A., von Wurstemberger, P.: Overcoming the curse of dimensionality in the numerical approximation of semilinear parabolic partial differential equations. Proc. Roy. Soc. A 476(2244), 20190630 (2020)

34. Hutzenthaler, M., Jentzen, A., von Wurstemberger, P.: Overcoming the curse of dimensionality in the approximative pricing of financial derivatives with default risks. Electron. J. Probab. 25, (2020)

35. Hutzenthaler, M., Kruse, T.: Multilevel Picard approximations of high-dimensional semilinear parabolic differential equations with gradient-dependent nonlinearities. SIAM J. Numer. Anal. 58(2), 929-961 (2020)

36. Karatzas, I., Shreve, S.E.: Brownian motion and stochastic calculus, second ed., vol. 113 of Graduate Texts in Mathematics. Springer-Verlag, New York (1991)

37. Kebaier, A.: Statistical Romberg extrapolation: a new variance reduction method and applications to option pricing. Ann. Appl. Probab. 15(4), 2681-2705 (2005)

38. Kloeden, P.E., Platen, E.: Numerical solution of stochastic differential equations, vol. 23 of applications of mathematics (New York), p. 632. Springer-Verlag, Berlin (1992)

39. Maruyama, G.: Continuous Markov processes and stochastic equations. Rend. Circ. Mat. Palermo (2) 4, 48-90 (1955)

40. Pardoux, É., Peng, S.: Backward., stochastic differential equations and quasilinear parabolic partial differential equations. In Stochastic partial differential equations and their applications (Charlotte, NC, : vol. 176 of Lecture Notes in Control and Inform. Sci. Springer, Berlin 1992, 200-217 (1991)

41. Pardoux, É., Peng, S.G.: Adapted solution of a backward stochastic differential equation. Syst. Control Lett. 14(1), 55-61 (1990)

42. Peng, S.G.: Probabilistic interpretation for systems of quasilinear parabolic partial differential equations. Stochastics Stochastics Rep. 37(1-2), 61-74 (1991)

43. Prévôt, C., Röckner, M.: A concise course on stochastic partial differential equations, vol. 1905 of Lecture Notes in Mathematics. Springer, Berlin. 144 pages (2007)

44. Robbins, H.: A remark on stirling's formula. Am. Math. Monthly 62(1), 26-29 (1955)

45. Skorohod, A.V.: Branching diffusion processes. Teor. Verojatnost. i Primenen. 9, $492-497$ (1964)

46. Yong, J., Zhou, X. Y.: Stochastic controls, vol. 43 of Applications of Mathematics (New York). SpringerVerlag, New York, (1999). Hamiltonian systems and HJB equations

47. Zhang, J.: A numerical scheme for BSDEs. Ann. Appl. Probab. 14(1), 459-488 (2004)

\title{
Affiliations
}

\section{Weinan $E^{1}(D) \cdot$ Martin Hutzenthaler ${ }^{2} \cdot$ Arnulf Jentzen $^{3,4,5}$ (D) Thomas Kruse $^{6}$}

\author{
Weinan E \\ weinan@math.princeton.edu \\ Martin Hutzenthaler \\ martin.hutzenthaler@uni-due.de \\ Thomas Kruse \\ thomas.kruse@math.uni-giessen.de
}

1 Department of Mathematics and Program in Applied and Computational Mathematics, Princeton University, Princeton, NJ 08544-1000, USA

2 Faculty of Mathematics, University of Duisburg-Essen, 45117 Essen, Germany

3 Seminar for Applied Mathematics, Department of Mathematics, ETH Zurich, 8092 Zurich, Switzerland 
4 Applied Mathematics: Institute for Analysis and Numerics, Faculty of Mathematics and Computer Science, University of Münster, 48149 Munster, Germany

5 School of Data Science and Shenzhen Research Institute of Big Data, The Chinese University of Hong Kong, Shenzhen 518172, China

6 Institute of Mathematics, University of Gießen, 35392 Giessen, Germany 